\title{
Metabolic engineering of Saccharomyces cerevisiae for efficient conversions of glycerol to ethanol
}

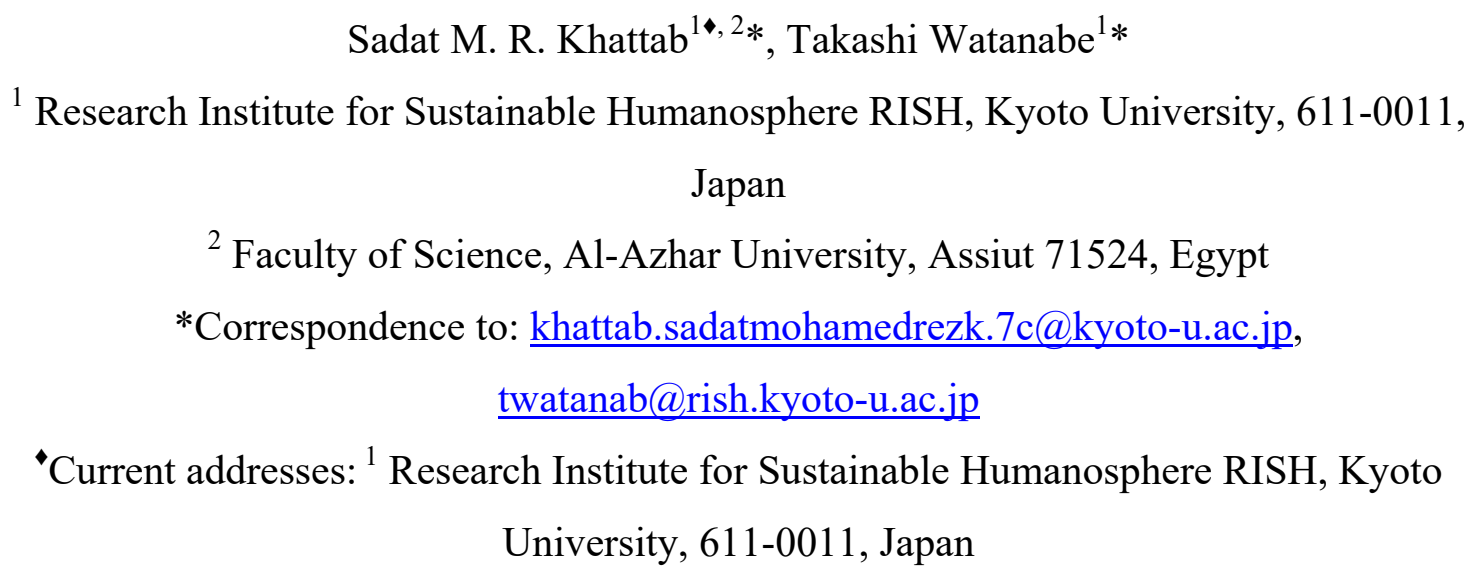

Glycerol is an eco-friendly solvent enhancing plant-biomass decomposition through the glycell process to bio-based chemicals. Nonetheless, the lack of efficient conversion of glycerol by natural Saccharomyces cerevisiae restrains many biorefineries-scenarios. Here, we outline a comprehensive strategy for generating efficient glycerol fermenting $S$. cerevisiae via rewriting the oxidation of cytosolic nicotinamide adenine dinucleotide by $\mathrm{O}_{2}$-dependent dynamic shuttle while abolishing glycerol phosphorylation and biosynthesis pathways. By following a vigorous glycerol oxidative pathway, our engineered strain demonstrated a breakthrough in conversion efficiency $(\mathrm{CE})$, reaching up to $0.49 \mathrm{~g}$-ethanol/g-glycerol $-98 \%$ of theoretical conversion - with production rate $>1$ $\mathrm{gL}^{-1} \mathrm{~h}^{-1}$ on rich-medium. Interestingly, the glycerol consumption and its fermentation unrepressed during the mixing by glucose until the strain produced $>86 \mathrm{~g} / \mathrm{L}$ of bioethanol with $92.8 \%$ of $\mathrm{CE}$. Moreover, fine-tuning of $\mathrm{O}_{2}$ boosted the production rate to $>2 \mathrm{gL}^{-1} \mathrm{~h}^{-1}$ with $82 \%$ of CE. Impressively, the strategy flipped the ancestral yeast even from nongrowing on glycerol, on the minimal medium, to a fermenting strain with productivities $0.25-0.5 \mathrm{gL}^{-1} \mathrm{~h}^{-1}$ and $84-78 \%$ of $\mathrm{CE}$, respectively. Our findings promote utlising glycerol efficiently in several eco-friendly biorefinery approaches.

Keywords: Saccharomyces cerevisiae, bioethanol, glycerol fermentation, metabolic engineering, rewriting cofactors

Summary: Efficient fermentation of glycerol in $S$. cerevisiae was established by 1 comprehensive engineering of glycerol pathways and rewriting NADH pathway. 
One of the challenges for sustaining the future humanosphere is the production of adequate bio-based chemicals and fuels from renewable resources with the goal of reducing greenhouse gas emissions. The paradigm of using scientific advances with metabolic engineering and biotechnology to meet the emerging needs for biofuels, as well as related materials and chemicals, has been envisioned and created on a commodity scale (Lovins et al, 2004; Ragauskas et al, 2006; Meadows et al, 2016). A massive requirement for ethanol recently arose for use in sanitisers because of the COVID-19 pandemic, as a concentration of $62 \%-71 \%$ ethanol has been demonstrated to deactivate virus particles on the skin and those that persist on inanimate surfaces such as metal, glass, and plastic (Kampf et al, 2020). Bakers' yeast (Saccharomyces cerevisiae) has several desirable characteristics for bioethanol production, such as long and safe history of use, unicellular structure, short life cycle, remarkable fermentation abilities, robustness against inhibitors, stress tolerance during industrial levels of production, the existence of a global infrastructure for production of bioethanol from starch and molasses, and availability of tools for genetic recombination. In addition, bakers' yeast is subject to adaptive evolution or even hybridisation and hence has been selected as a top model platform of microbial cell factories for several biotechnological applications (Peris et al, 2017; Khattab \& Watanabe, 2019; Xiberras et al, 2019). The first global production of bioethanol was successfully established for blending with gasoline as a transportation biofuel. Owing to environmental, political, security and bio-economic reasons, the demand for bioethanol is increasing. However, the resources required for fermentation are limited, and challenges exist to overcome the drawbacks of second- and third-generation applications of bioethanol from lignocellulosic biomass and algae through evolving the maximum efficiencies in ethanol production from xylose and acetic acid with glucose (Luque et al, 2008; Deepak \& Gregory, 2011; Khattab et al, 2013; Wei et al, 2013; Khattab \& Kodaki, 2014).

In the past decade, glycerol-producing industries, particularly that of biodiesel, have expanded and accumulated substantial quantities of glycerol, which has led to price drops (Nomanbhay et al, 2018). Although the reduction degree of glycerol $\left(\mathrm{C}_{3} \mathrm{H}_{8} \mathrm{O}_{3}\right)$ is higher than that of other fermentable sugars (Yazdani \& Gonzalez, 2007), glycerol is 
63 classified as a non-fermentable carbon in native S. cerevisiae (Xiberras et al, 2019). Additionally, glycerol is a carbon source poorly utilised primarily via the glycerol-3phosphate pathway (herein referred to as the G3P pathway), which is composed of glycerol kinase (GUT1) and FAD-dependent-mitochondrial glycerol-3-phosphate dehydrogenase (GUT2) (Sprague \& Cronan, 1977). Yeast biosynthesis glycerol for mitigating osmotic stress and optimising the redox balance (Ansell et al, 1997). Furthermore, glycerol catabolism is subject to the repression and transcriptional regulation of glucose through respiratory factors and the GUT1 and GUT2 genes (Grauslund et al, 1999; Grauslund \& Ronnow, 2000; Roberts \& Hudson, 2009; Turcotte et al, 2010). The importance of glycerol as a carbon source that can be utilised by yeast cells has been recognised and prompted a study of the relationship between molecular inheritance and the physiology of glycerol uptake and its metabolism. The study revealed intraspecies diversity ranging from good glycerol grower to non-growers among $52 \mathrm{~S}$. cerevisiae strains on a synthetic medium without supporting supplements and showed that the glycerol growth phenotype is a quantitative trait (Swinnen et al, 2013). The study confirmed that GUT1 is one of the genetic loci that share a glycerol growth phenotype in a particular good glycerol grower, the haploid segregant CBS 6412-13A (Swinnen et al, 2013). Two further superior alleles of cytoplasmic ubiquitin protein ligase E3 (UBR2) and cytoplasmic phosphorelay intermediate osmosensor and regulator (SSK1) were found to link with GUT1 for growth on synthetic medium without supporting supplements (Swinnen et al, 2016). These pivotal roles of UBR2 and GUT1 during glycerol assimilation by yeast were further confirmed by another study that resequenced the whole genomes of glycerol-evolved strains (Ochoa-Estopier et al, 2011; Ho et al, 2017). Although the G3P pathway has been evidenced as being the main catabolic pathway for glycerol catabolism in S. cerevisiae, its heterologous replacement with the glycerol oxidative pathway (DHA; dihydroxyacetone pathway) combined with the glycerol facilitator (FPS1) resulted in the restoration of growth characteristics like those of the parental strain. Furthermore, this replacement in a negative glycerol grower strain bearing the swapped UBR2 $2_{\mathrm{CBS} 6412-13 \mathrm{~A}}$ allele stimulated growth to the highest specific growth rate ever reported on glycerol synthetic media (Klein et al, 2016). 
Using an approach to produce 1,2-propanediol from glycerol, a significant amount of ethanol $(18 \mathrm{~g} / \mathrm{L})$ had accumulated during the first day as a byproduct, particularly on rich media (Islam et al, 2017). That study for production of 1,2-propanediol addressed a metabolic engineering strategy combined with heterologous replacement of the G3P route by the DHA-FPS pathway (Klein et al, 2016) with a module to produce 1,2-propanediol and underexpression of the triosephosphate isomerase gene (TPI1) (Islam et al, 2017). Limiting oxygen availability in shaking flask cultures resulted in increased production of ethanol from glycerol [0.165g-ethanol/g-glycerol $\left(\mathrm{g}^{\mathrm{e}} / \mathrm{g}^{\mathrm{g}}\right)$ compared with $\left.0.344 \mathrm{~g}^{\mathrm{e}} / \mathrm{g}^{\mathrm{g}}\right]$, with production rates ranged from 0.11 to $0.18 \mathrm{gL}^{-1} \mathrm{~h}^{-1}$ on buffered synthetic medium, for facilitating the understanding the engineering of valuable products more reduced than ethanol (Aßkamp et al, 2019) using genetic modifications of heterologous replacement of the G3P route by the DHA-FPS pathway (Klein et al, 2016). It is worth emphasising that glycerol has been considered as a non-fermentable carbon source in S. cerevisiae (Xiberras et al, 2019), and attempts have been made to ferment glycerol in this yeast. These experiments were initiated by overexpressing a native DHA pathway including the glycerol dehydrogenase ScGCY1 and dihydroxyacetone kinase (DAK) to produce $0.12 \mathrm{~g}^{\mathrm{e}} / \mathrm{g}^{\mathrm{g}}$ with a production rate of $0.025 \mathrm{gL}^{-1} \mathrm{~h}^{-1}(\mathrm{Yu}$ et al, 2010). The methylotrophic yeast Ogataea polymorpha has been tested for bioethanol production from glycerol by overexpressing the genes involved in either the DHA or G3P pathways with integration of the gene for the glycerol transporter FPS1 from Pichia pastoris. Furthermore, the recipient strain exhibited overexpression of the genes encoding pyruvate decarboxylase $(P D C) 1$ and alcohol dehydrogenase $(A D H)$ 1. Nonetheless, overall ethanol production was relatively low (10.7 $\mathrm{g}$ ethanol as maximum accumulated product and $0.132 \mathrm{~g}^{\mathrm{e}} / \mathrm{g}^{\mathrm{g}}$ ) (Semkiv et al, 2019). To date, there is no known native or genetically engineered yeast strain that can convert glycerol efficiently to ethanol.

Of note, we previously developed a novel biomass pretreatment method employing the glycell process with the catalysis of alum $\left[\mathrm{AlK}\left(\mathrm{SO}_{4}\right)_{2}\right]$, with additional enhancement via microwave (Ohashi \& Watanabe, 2018) There is a need for developing a Saccharomyces cerevisiae capable of efficiently fermenting glycerol following glycerolysis of biomass. Such glycerol-fermenting strain will co-ferment glycerol and synergist the ethanol production from hydrolysed lignocellulosic biomass. In the present 
124 study, we report the details of modelling the yeast cell to redirect glycerol traffic to

125 bioethanol production to highest ever reported $>8.6 \%$ - even in the presence of glucose-

126 through innovation of the forthcoming systematic metabolic engineering processes

127 outlined in Figure 1 as follows: 1) abolishment of the inherent glycerol biosynthesis

128 pathway by knocking out NAD-dependent glycerol-3-phosphate dehydrogenase (GPD) 1

129 and retaining the second isoform of ScGPD2 for requirements of glycerol-3-phosphate

130 for lipid metabolism; 2) replacement of cytosolic NADH oxidation through the ScGPD1

131 shuttle with a more effective $\mathrm{O}_{2}$-dependent dynamic shuttle of water forming the NADH

132 oxidase NoxE to replenish $\mathrm{NAD}^{+}$for the integrated gene of glycerol dehydrogenase

$133(O p G D H)$; 3) knocking out the first gene of the G3P pathway (GUT1); and 4)

134 implementing a vigorous oxidative pathway via overexpression of two copies of both the

135 heterologous genes of glycerol dehydrogenase from $O$. polymorpha $(O p G D H)$ and the

136 glycerol facilitator CUFPS1, besides two copies of both the endogenous genes ScTPI1

137 and ScDAK1 with one copy of ScDAK2.

138 Results

139 Systematic metabolic engineering

140 Vigorous glycerol dehydrogenase is an essential initiator of glycerol fermentation

141 Initial verification of overexpression of $O p G D H$ (Yamada-Onodera et al, 2002) in

142 S. cerevisiae strain D452-2 showed strong effects compared with that of the native

143 ScGCY1 gene alone, or even when ScGCY1 was integrated with other endogenous

144 oxidative pathway genes (glycerol proton symporter of the plasma membrane ScSTL1,

$145 S c D A K 1, S c D A K 2$ and ScTPI1) in the recombinant strain GF2 (Table 1). The specific

146 activity of ScGCY1 in GF2 is negligible at that studied condition compared with $O p G D H$

147 (Table 2 and the additional supplementary data). Although, the GF2 strain has a higher

148 enzyme activity in both ScDAK and triosephosphate isomerase (ScTPI1) by 64 and 17\%,

149 respectively, compared with the strain harbouring the GDH gene (Table.2). As a result of

150 the higher activity of $O p G D H$, the recombinant yeast, which was named GDH, consumed

151 glycerol faster than GF2 in micro-aerobic condition, with a $21 \%$ increase in ethanol

152 production, which was only $10 \%$ in GF2 as compared with that in the parental strain (Fig.

153 2). With semi-aerobic fermentation (10:100 medium: flask volume) using mixed glucose 
154 and glycerol for the GDH strain, improved glycerol consumption and ethanol production

155 from $25 \%$ to $40 \%$ and $21 \%$ to $64 \%$, respectively, before switching to the re-utilisation of

156 ethanol when compared with previous micro-aerobic conditions (20:100 medium: flask

157 volume) (Fig. 2 and 3). These results indicate that the first step for efficient glycerol

158 fermentation should be through an effective GDH gene, initiated here with the action of

$159 \mathrm{OpGDH}$. Furthermore, we confirmed that glycerol consumption occurred through the

160 constructed DHA pathway, in which its consumption was not significantly decreased

161 after knockout of ScGUT1, the first gene in the G3P pathway (Fig. 2). Notably, activating

162 the genes of the G3P pathway (i.e. ScSTL1, ScGUT1, ScGUT2 and ScTPI1) in the

163 recombinant strain GA2 (Table 1) did not result in significant improvement in the ethanol

164 production (Fig. 2).

\section{Efficient rewriting of the NADH oxidation pathway in $S$. cerevisiae by an $\mathrm{O}_{2}$ -} dependent dynamic water shuttle forming LINoxE to replace ScGPD1

We comprehensively studied the replacement of GPD shuttles by waterforming NADH oxidase from Lactococcus lactis subsp. lactis (LINoxE). The GPD shuttle is the first step in glycerol biosynthesis and represents one of the well-known systems for renovating cytosolic $\mathrm{NAD}^{+}$from $\mathrm{NADH}$ produced during the metabolic process, such as those from the oxidation of glyceraldehyde-3-phosphate (GA3P). Consequently, glycerol is secreted upon increase of cytosolic NADH for replenishment of $\mathrm{NAD}^{+}$. Therefore, we first rated the participation levels of ScGPD1 and ScGPD2 in glycerol biosynthesis in our ancestral strain D452-2 at 10\% glucose fermentation by deleting each isoform separately. In this stage, glycerol did not add to prevent interference with the biosynthesised glycerol. Besides, inducing the osmostress by the supplemented glucose at $10 \%$ is another factor for activating GPD shuttles. The data revealed that the participation ratio of ScGPD1 in glycerol biosynthesis was $82 \%$, in which glycerol secretion from $\triangle S c G P D 1$ was ratio compared to WT of $0.47 \mathrm{~g} / 2.56 \mathrm{~g}$ and $23 \%$, where $2.08 \mathrm{~g}$ glycerol produced from $\triangle$ ScGPD2 (Table 3; Fig. S1a). Replacing ScGPD1 with LINoxE reduced glycerol secretion by $98 \%$, in which 0.14 $\mathrm{g} / 2.56 \mathrm{~g}$ of WT glycerol was secreted, whereas it only reduced to $29 \%$ for the NoxE/GPD2 strain, in which $1.82 \mathrm{~g} / 2.56 \mathrm{~g}$ of WT was secreted (Table 3; Fig.S1a). On 
184 the other hand, replacing both SCGPD1 and ScGPD2 with LINoxE not only prevented

185 glycerol formation but also reduced glucose consumption significantly and obstructed

186 cell growth and fermentation by almost the same levels (at 15\%) while increasing 187 secretion of acetic acid by 2.46 -fold (Table 3; Fig.S1a-e). Similarly, replacing ScGPD1

188 by LINoxE while deleting ScGPD2 had harmful effects on fermentation speed and 189 products (Table 3; Fig. S1a-e). Comparatively, the overexpressed LINoxE gene in the

190 URA3 locus with the conserved native activity of the glycerol biosynthesis pathway 191 exhibited a moderate reduction in glycerol production of only $41 \%(1.53 \mathrm{~g} / 2.56 \mathrm{~g})$ in the 192 D452-2 strain (Table 3; Fig. S1a). Notably, replacing GPD with LINoxE altered glycerol 193 production to result in an increase in acetic acid production (Table 3; Fig. S1b). The 194 efficiency of replacing ScGPD1, as an essential shuttle for oxidising the cytosolic $195 \mathrm{NADH}$, by water-forming $\mathrm{NADH}$ oxidase was confirmed by estimating the NADH 196 concentration, where its concentration decreased by over 4 times compared with the 197 ancestor strain (Table 2). Exclusively replacing ScGPD1 with LINoxE is an excellent 198 approach to eliminating glycerol formation during glucose fermentation to ethanol under 199 the studied condition, where ethanol production increased by $9 \%(0.474 / 0.432 \mathrm{~g}$-ethanol 200 /g-glucose; Table 3), therefore, consolidating this replacement with GDH strain could improve the efficiency of glycerol conversion to ethanol.

\section{Integrating $G D H$ and NoxE with $\triangle S c G P D 1$}

Based on previous results from the recombinant GDH strain and the data for replacement of oxidising shuttles of cytosolic NADH by LINoxE, we studied the recycling outputs of $\mathrm{NAD}^{+} / \mathrm{NADH}$ between $G D H$ and LINoxE genes. In addition to such recycling, deleting ScGPD1 during substitution with LINoxE abolishes glycerol formation and decreases ramification of DHAP, which consolidates straightforward progression to the glycolysis route and ethanol formation. Thus, we engineered a strain that combined GDH and NOXE (Table 1). This round of recombination (GDH-NOXE or GN strain) was tested for its ability to ferment glycerol in comparison with GDH, NOXE and wild type strains. This innovative integration clearly showed improvements in both efficiency of glycerol conversion to ethanol and delay in the time of cell reprogramming to utilise the produced ethanol. After $6 \mathrm{~h}$ both ancestral strains and the 
214 engineered NOXE strain started to re-utilise the ethanol produced from glucose

215 without further significant consumption of glycerol, in which the maximum ethanol 216 produced was $4.7 \mathrm{~g} / \mathrm{L}$ (Fig. 3). In the GDH strain, the time for reusing the produced

217 ethanol was delayed to $26 \mathrm{~h}$ with increases in ethanol production to $11.82 \mathrm{~g} / \mathrm{L}$, 218 representing $0.27 \mathrm{~g}$-ethanol/g-glucose-glycerol. In the case of GN strain, integration not 219 only boosted ethanol production to $13.27 \mathrm{~g} / \mathrm{L}(0.31$ g-ethanol/g-glucose-glycerol $)$ at $22026 \mathrm{~h}$, but also extended the fermentation time to $32 \mathrm{~h}$ and further increased production 221 of ethanol to $14.42 \mathrm{~g} / \mathrm{L}$ before the switching to consume this ethanol (Fig. 3). In 222 addition to the substantial activity of glycerol dehydrogenase in both strain GDH and $223 \mathrm{GN}$, a cofactor ratio $\left(\mathrm{NADH} / \mathrm{NAD}^{+}\right)$showed a significant change from 0.68 to 0.99 224 between GDH and GN strains, respectively (Table.2). This increase in the concentration of NADH is pointing to the higher efficiency of replacing ScGPD1 by LINoxE shuttle, regardless that cells have cultivated at micro-aerobic conditions (10:50 227 medium: falcon tubes volume).

\section{Overexpressing other DHA pathway genes: TPI1, DAK1, DAK2 and FPS1}

Although clear impacts were observed for recycled inputs within the previous recombination, we deduced further limitations in the activity of other genes in the DHA pathway-TPI1, DAKs and FPS1-which affect the full traffic of glycerol conversion to ethanol. In this point, we must clarify that ScDAK2 and ScTPI1 was not considered during the previous studies of converting glycerol to ethanol. Therefore, we proceeded to overexpress the whole genes included in the DHA pathway at this stage of systematic engineering. A promoter phosphoglycerate kinase (PGK) with its terminator was used to activate the endogenous genes ScTPI1, ScDAK1 and ScDAK2. The glycerol facilitator gene from Candida utilis (CuFPS1) (Klein et al, 2016) was heterologously expressed under the control of PGK promoter and the ribosomal 60S subunit protein L41B (RPL41B) terminator. The previous recombinant strain GN has been used as competent cells for receiving this one set of genes in the AUR-1C locus to generate a new strain, which was termed GN-FDT (Table 1). In the GN-FDT strain, the specific enzyme activity of $O p G D H$ increased by $9 \%$ compared with the GN strain. Also, ScDAK and ScTPI1 increased by $83 \%$ and $16 \%$, respectively, compared with the 
244 WT strain. Moreover, the NADH/NAD ${ }^{+}$ratio decreased to $0.33 \%$ of the GN (Table 2).

245 This fourth recombination step (GN-FDT strain) unequivocally solved one of the main

246 problems in this study, in which switching to ethanol utilisation was prevented before

247 the full consumption of glycerol. The consumption rate reached $1 \mathrm{gL}^{-1} \mathrm{~h}^{-1}$ and produced

$24820.95 \mathrm{~g} / \mathrm{L}$ of ethanol using this recombinant strain. Nonetheless, the conversion

249 efficiency of ethanol production appeared to be less than $48 \%$ of its theoretical value 250 (Fig. 3).

\section{Super-expressing DHA pathway using a further copy of ScTPI1, ScDAK1,}

The results from the fourth step of genetic engineering performed here (GNFDT strain) highlighted the effect of the limited activities of all the genes of the DHA pathway on improving productivity with the possibility to strengthen the efficiencies by another copy of the pathway. We carefully selected and designed the strongest expression systems that were not likely to be affected by regulator repressors to constitutively express this assortment of genes (Ito et al, 2013; Yamanishi et al, 2013; Ito et al, 2016; Wei et al, 2017; Nambu-Nishida et al, 2018). By use of the Gibson hybrid assembly and PCR we constructed one module named M1 (Fig. S2) with the following expression systems: TEF1 promoter-CYC1 terminator, TYS1 promoterATP15 terminator, TDH3 promoter-mutated d22DIT1 terminator and FBA1 promoterTDH3 terminator, for the genes CuFPS1, OpGDH, ScDAK1 and ScTPI1, respectively. Therefore, we intensified an entire glycerol oxidation pathway by integrating additional copies of the genes CuFPS1, OpGDH, ScDAK1 and ScTPI1 during the replacement of GUT1-which abolished the G3P pathway-while attempting to overcome previous inadequacies during this stage of recombination. As a result, the specific enzyme activities of $O p G D H, S c D A K$ and ScTPI1 were further enhanced by $56 \%, 256 \%$ and $13 \%$, respectively, compared with the GN-FDT strain. Besides, the $\mathrm{NADH} / \mathrm{NAD}^{+}$ratio decreased to 0.11 (Table 2). Interestingly, we obtained unique findings in this fourth step of recombination (SK-FGG) for glycerol consumption and ethanol production that were not previously reported in any safe organism with the developed strain GN-FDT-M1, named SK-FGG (Table 1, Fig. 3). The consumption 
274 rate of this strain reached $2.6 \mathrm{gL}^{-1} \mathrm{~h}^{-1}$ from glycerol at the described experimental

275 conditions, and the productivity paced $1.38 \mathrm{gL}^{-1} \mathrm{~h}^{-1}$ of ethanol with a conversion

276 efficiency of 0.44 g-ethanol/g-glucose-glycerol (Fig. 3).

277 Fermentation characteristics at high initial concentrations of glycerol, with and without adding glucose, or with the higher availability of oxygen

With the current state of metabolic engineering, we examined fermentation characteristics at higher initial concentrations of glycerol $(110 \mathrm{~g} / \mathrm{L})$ in the absence and presence of glucose $(22.5 \mathrm{~g} / \mathrm{L})$, where glucose was reported as a suppressor for glycerol fermentation (Table $4[\mathrm{~A} \& \mathrm{~B}])$. We also tested fermenting a further fedbatching of $100 \mathrm{~g} / \mathrm{L}$ glycerol to the condition of [B] in (Table 4 [C]). To further confirming the engineered strain is unsubjected to repression by glucose during the glycerol fermentation, we lifted the glucose level to $45 \mathrm{~g} / \mathrm{L}$ with decreased the glycerol concentration by $25 \%$ to $82 \mathrm{~g} / \mathrm{L}$ before further adding $100 \mathrm{~g} / \mathrm{L}$ glycerol as a fed-batch (Table 4 [D]). These outlines also testing the strain SK-FGG to produce an economically distillable ethanol titer (Table 4 [D]). The concentrations of glucose and glycerol in [D] are relatively like those obtained after the glycell process (Ohashi \& Watanabe, 2018). Finally, we tested the higher oxygen availability on the production rate and efficiency at $90 \mathrm{~g} / \mathrm{L}$ glycerol without glucose. The strain SK-FGG exhibited outstanding performance in micro-aerobic conditions at a higher initial concentration

293 of glycerol-glucose mixture in YP medium, where its conversion efficiency reached $98 \%\left(0.49 \mathrm{~g}^{\mathrm{e}} / \mathrm{g}^{\mathrm{g}}\right)$ with a production rate of $>1 \mathrm{gL}^{-1} \mathrm{~h}^{-1}$ of ethanol after consumed 82.5 $\mathrm{g} / \mathrm{L}$ of glycerol from one fed-batch condition (Table 4, condition [A]). Acetic acid accumulated at $1.14 \mathrm{~g} / \mathrm{L}$ at this condition (Table 4, condition [A]). Even at mixing glycerol with $22.55 \mathrm{~g} / \mathrm{L}$ of glucose, its conversion efficiency was comparatively the same (Table 4, condition [B]). Interestingly, the strain engineered here is exceptional in its capacity to harmonise fermenting glycerol with glucose, along with an accumulation of $>86 \mathrm{~g} / \mathrm{L}$ of bioethanol with additional fed batching of glycerol (Table 4, [D]). With higher initial glucose at condition [D], cell density was promoted by $31 \%$ compared with the case [C]; besides, a minor reduction of the efficiency of ethanol conversion (Table 4, [C \& D]). Notably, increasing oxygen availability by increasing 
304 flask volume by 2.5-fold while keeping broth volume constant remarkably accelerated

305 glycerol consumption to $>5 \mathrm{gL}^{-1} \mathrm{~h}^{-1}$ (Table 4, [E]). In addition, the rate of ethanol

306 production increased to $>2 \mathrm{~g} \mathrm{gL}^{-1} \mathrm{~h}^{-1}$. However, its conversion efficiency dropped to

$30782.8 \%$ of the theoretical value (Table $4,[\mathrm{E}]$ ).

\section{Ferment glycerol as a sole carbon source}

Our ancestor strain, D452-2 is MATa, leu2, his3, ura3, can1 genetic base (Hosaka et al, 1992), and even after supplement uracil, leucine, and histidine to minimal medium, it is growthless on glycerol as a sole carbon (Fig.S4). To verify the efficiency of the best-engineered strain during this study, SK-FGG, glycerol was tested for fermentation as the sole carbon when supplemented into a yeast nitrogen base (YNB) medium with $20 \mathrm{mg} / \mathrm{L}$ of leucine and histidine. Testing was performed at four different oxygen availabilities. Under the strict anaerobic conditions, there was no further growth and no production of ethanol (Table 5). Under micro-aerobic conditions (20 $\mathrm{ml}$ of culture in a $100-\mathrm{ml}$ Erlenmeyer flask at $30^{\circ} \mathrm{C}$ with shaking at $200 \mathrm{rpm}$ ), $37.17 \mathrm{~g}$ of glycerol was consumed with a consumption rate of $0.62 \mathrm{gL}^{-1} \mathrm{~h}^{-1}$ and a production rate of $0.25 \mathrm{gL}^{-1} \mathrm{~h}^{-1}$. The efficiency of ethanol conversion reached $0.42 \mathrm{~g} / \mathrm{g}$ glycerol. Acetic acid accumulated at $0.78 \mathrm{~g} / \mathrm{L}$ in this condition (Table 5). Glycerol was consumed more rapidly in the semi-aerobic condition $(20 \mathrm{ml}$ of culture in a $200-\mathrm{ml}$ Erlenmeyer flask). The consumption rate was $>1 \mathrm{gL}^{-1} \mathrm{~h}^{-1}$, which raised the ethanol production rate to $0.44 \mathrm{gL}^{-1} \mathrm{~h}^{-1}$. There was $2.88 \mathrm{~g}$ of acetic acid cumulation in this condition. As a result, the total conversion reached $0.44 \mathrm{~g} / \mathrm{g}$ glycerol (Table 5). With further elevation of the oxygen availability in 20:300-ml liquid medium: flask volume ratio, the rate of glycerol consumption and ethanol production rate was boosted to 1.29 and $0.5 \mathrm{gL}^{-1} \mathrm{~h}^{-1}$, respectively. The efficiency of ethanol conversion was $0.39 \mathrm{~g}^{\mathrm{e}} / \mathrm{g}^{\mathrm{g}}$, and the total convertibility was $0.45 \mathrm{~g}^{\mathrm{e}} / \mathrm{g}^{\mathrm{g}}$, which represents $90 \%$ of the theoretical conversion regardless of the utilised glycerol in cell formation (Table 5).

\section{Discussion}

Recently, microbial technologies for exploiting glycerol as a carbon source for producing valuable products have gained increasing attention, where a considerable 
amount of glycerol accumulates as an unavoidable byproduct from the expansion of biodiesel industries. Moreover, we have developed a novel promising pretreatment method using glycerol for support the solvolesis of lignocellulosic biomass with alum and microwaving (Ohashi \& Watanabe, 2018). Therefore, generating a yeast strain capable of converting such a mixture of glucose and xylose with glycerol following glycerolysis was inevitable for developing our methodology. Here we describe the initial study to construct a novel strain that can efficiently convert pure glycerol with glucose to ethanol.

It is well known that native $S$. cerevisiae contains full genes for two metabolic pathways (DHA and G3P) for glycerol catabolism (Fig. 1); nonetheless, glycerol was considered as non-fermentable and an unfavourable carbon source as a feedstock (Sprague \& Cronan 1977; Xiberras et al, 2019). Furthermore, one of the ancestral of our laboratory strain belongs to S288C (Kodaki \& Yamashita 1989; Hosaka et al,1990; Hosaka et al,1992) (Table 1 and Fig. S4) that has been classified as a negative glycerol grower on synthetic medium (Swinnen et al, 2013). Therefore, we tested our strain D4522 for growth on glycerol as a sole carbon source in YNB medium supplemented by 20 $\mathrm{mg} / \mathrm{L}$ of leucine, histidine and $5 \mathrm{mg} / \mathrm{L}$ of uracil. The maximum optical density obtained after the growing for $180 \mathrm{~h}$ was $(0.28)$. That data evidences the classification of the ancestral D452-2 strain in a negative glycerol grower class (Fig. S4). Hence, herein we initially focused on using a conventional YP medium supplemented glycerol or/and glucose to overcome the limiting nutritional factors that arise from using a synthetic or minimum medium, as well as the necessity for providing amino acids and nucleic bases. Moreover, this YP medium showed enhancement in bioethanol accumulation from glycerol during the onset of 1, 2-propanediol production (Islam et al, 2017).

Although abolishing SCTPI1 has been a pivotal hub to produce glycerol from glucose (Overkamp et al, 2002), which was reversed here, this gene has not been integrated with a previous study examining overexpressing the native DHA-pathway (Yu et al, 2010). Therefore, we initiated our study by updating that work (Yu et al, 2010) via combination of overexpression of ScTPI1 and ScDAK2 with the DHA pathway and replaced a previously reported GUP gene with a currently unique and generally accepted glycerol transporter SCSTL1 to track restrictions in the native oxidative pathway during glycerol fermentation. Hence, we constructed the strain GF2, which overexpressed the 
genes ScSTL1, ScGCY1, ScDAK1, ScDAK2 and ScTPI1. Additionally, we constructed the GCY strain, which overexpressed only the ScGCY1 gene and GF1, which activated the genes ScGCY1, ScDAK1, ScDAK2. Similarly, we generated GA1, which overexpressed ScGUT1, ScGUT2 and ScTPI1, as well as GA2, which activated ScSTL1, ScGUT1, ScGUT2 and ScTPI1.

Albeit we detected a significantly faster growth rate, particularly for GF2 (Fig. S4), we did not observe a significant improvement in ethanol production with that strain overexpressed its ScGCY1 only at the conditions tested in Fig. 2 (data omitted to avoid overlap with the ancestral strain). However, we detected significant ethanol production by GA2 and GF2 that was rapidly re-consumed in the presence of higher concentrations of glycerol (Fig. 2). Concurrently, we recognised the limited activity of the endogenously overexpressed ScGCY1 gene compared with glycerol dehydrogenases from $O$. polymorpha (Nguyen \& Nevoigt, 2009). Therefore, we constructed a yeast harbouring $O p G D H$ named GDH to be tested with GF2 during glycerol fermentation. Furthermore, we confirmed the limited activity of the overexpressed ScGCY1 gene compared with glycerol dehydrogenases from $O$. polymorpha (Table 2). As a result of these comparative studies, such an active $O p G D H$ gene is the first key for deciphering glycerol fermentation, although the sole integration of $O p G D H$ was not enough to induce an efficient conversion (Fig. 2). On the other hand, overexpressing the native glycerol catabolic pathway G3P in the GA2 strain did not demonstrate promising results compared with this oxidative pathway. We hypothesise that this is a limit of the respiratory chain during glycerol consumption and thus, restricted the renovation of $\mathrm{FAD}^{+}$for converting glycerol-3-phosphate to DHAP through GUT2, considering that this phosphorylated G3P pathway is subject to repression and transcriptional regulation with the presence of glucose (Grauslund et al, 1999; Grauslund \& Ronnow, 2000; Roberts \& Hudson, 2009; Turcotte et al, 2010; Saito \& Posas, 2012; Babazadeh et al, 2017).

Besides the induction for expressing target genes, one of the other main obstacles affecting the efficiency of microbial production is to meet the stoichiometries of the engineered metabolic pathways, cofactors and ATP/oxygen ratios, especially for those pathways which require cofactors for their activation (Vemuri et al, 2007; Deepak \& Gregory, 2011; Meadows et al, 2016). As an integrated potent OpGDH in S. cerevisiae, 
395 we visualised that GPDs shuttles may promote activation of oxidation of the plethora

396 from cytosolic NADH (Table 2) and a reduction of DHAP into glycerol biosynthesis

397 pathway. Additionally, a ramification of glycerol-3-phosphate into glycerolipid pathway

398 may take place (Zheng \& Zou, 2001). Likewise, DHAP may be distributed into

399 phospholipid and methylglyoxal biosynthesis (Murata et al, 1985; Zheng \& Zou, 2001).

400 On the other hand, external mitochondrial NADH dehydrogenase (NDE) 1 showed

401 involvement in recycling the cofactor during glycerol metabolism (Aßkamp et al, 2019).

402 Furthermore, it is well known that the alcohol dehydrogenase $A D H 2$ primarily catalyses

403 the reaction of oxidising ethanol to acetaldehyde and the synthesis of $A D H 2$ is repressing

404 by glucose and vice versa in the presence of glycerol (Lutstorf et al, 1968). The activity

405 of $A D H$ within cells grown in glycerol yeast extract medium has been shown to be ten

406 times higher as compared to those cultured in glucose (Lutstorf et al, 1968). Further

407 investigations are needed to elucidate and quantify the participation of each of the five

408 isoforms of $A D H$ during switching and utilise an ethanol produced concomitant the 409 glycerol.

410 In native yeast with access to glucose, especially in the presence of oxygen, there 411 is a plethora of cytosolic NADH. As a result, there is a need for shuttles to re-oxidise this 412 surplus. The GPD shuttle plays an essential role in this regard with reduction of DHAP to 413 glycerol-3-phosphate to maintain this homeostasis (Larsson et al, 1998). Intracellular 414 redox homeostasis in $S$. cerevisiae comprises $>200$ reactions; thus, shuttles oxidising 415 NADH have been well studied (Ansell et al, 1997; Vemuri et al, 2007). One successful 416 strategy involves catalysing the oxidation of cytosolic NADH by heterologous expression 417 of a water-forming oxidase gene in S. cerevisiae for reducing the cytosolic NADH and 418 revert the glycerol biosynthesis from glucose to 2, 3-butanediol, and acetoin (Kim et al, 419 2015; Kim \& Hahn 2015; Bae et al, 2016; Kim et al, 2019). Also, the combination uses 420 NoxE and GPDs during the conversion of glucose to ethanol has been reported (Vemuri 421 et al, 2007). Nonetheless, there are no studies regarding the effects of replacing the native 422 shuttles of GPD with these alternative shuttles (NoxE) for oxidising cytosolic NADH 423 during ethanol production from glucose. Also, there are no reports about using NoxE 424 during the glycerol conversion to bioethanol. Therefore, in the second round of 425 recombination described here, we comprehensively focused on preventing that overflow 
426 to glycerol biosynthesis with the conservation of intracellular redox homeostasis during

427 glucose fermentation under micro-aerobic condition. Investigation of the nine constructed

428 strains of either deleted or replaced ScGPD1 and/or ScGPD2 by the LINoxE gene

429 indicated that replacing ScGPD1 by LINOXE is the best approach, in which glycerol

430 biosynthesis was effectively abolished by $98 \%$, and improvement in fermentation

431 efficiency by $9 \%$ was observed (Table 3 ). This strategy remarkably decreased cellular

432 NADH concentration by $76 \%$ under limited the oxygen availability (Table 2 ).

433 Expectedly, this single gene replacement will not exhibit significant progress 434 toward glycerol fermentation (Fig. 3). The low activity of native glycerol dehydrogenase 435 ScGCY1 is known (Nguyen \& Nevoigt, 2009). We confirmed there is no detectable 436 activity in our strain under that investigated conditions (Table 2). As detailed above, 437 ramification of DHAP represents another hindrance for straightforward progress into the 438 glycolysis route from glycerol. At this juncture, reduced circulation of DHAP into the 439 glycerol biosynthesis and G3P pathways was confirmed to be efficient for glycerol 440 fermentation by integrating this replacement of ScGPD1 by LINoxE within the GDH 441 strain. As expected, the strain harboured this unique point of integration (GDH-NOXE) 442 showed substantial improvement in ethanol production from glycerol, which reached $44328 \%$ compared with the GDH strain at the studied conditions, and was not considered for 444 other parameters, such as oxygen levels (Fig. 3). The role of abolishing ScGPD1 was 445 explicitly calculated from the data (Fig. 3), which represented $43 \%$ of that improved 446 ratio. The balance of cofactor was confirmed in this combination by measuring the 447 cellular content, as shown in Table 2. Utilising the recycled cofactors of NADH/NAD ${ }^{+}$ 448 for production of 1, 2-propanediol has been well studied during glycerol fermentation 449 (Islam et al, 2017).

The importance of activation of the other genes in the DHA pathway has been confirmed through continued bioethanol production until full glycerol consumption (Fig.3). Although we did not evaluate the effect of overexpressing each gene individually, we estimated the specific activities of OpGDH, ScDAKs, ScTPI1 in the recombinant strain and recognised the cooperative effects for overcoming that traditional and ambiguous phenomenon of re-consuming the produced ethanol earlier than the 
457 of ScDAKs plays another essential role with both OpGDH and ScTPI1 for glycerol

458 conversion, where GN-FDT showed higher specific enzyme activities

459 of OpGDH, ScDAKs, and ScTPI1 compared with GN and the ancestor strain (Table 2).

460 Moreover, conservation of the $\mathrm{NADH} / \mathrm{NAD}^{+}$at the lower ratios is substantial for the

461 straightforward reaction toward the ethanol formation. In this regard, it was reported that

462 the permeability of the three-carbon compounds, including glycerol in Candida utilis, is

463 much more rapid than that in bakers' yeast, which supports the efficient utilisation of

464 glycerol even at low concentrations (Gancedo et al, 1968). Therefore, we heterologously

465 expressed CUFPS1 in S. cerevisiae to support the influxes of glycerol in our strain as also

466 reported previously (Klein et al, 2016; Islam et al, 2017). Besides, ScDAK1 and ScDAK2

467 were characterised for detoxifying DHA, with $\mathrm{Km}_{(\mathrm{DHA})}$ of 22 and $5 \mu \mathrm{M}$ and $\mathrm{Km}_{(\mathrm{ATP})}$ of

$468 \quad 0.5$ and $0.1 \mathrm{mM}$, respectively (Molin et al, 2003), thus overexpressing ScDAK2 (which

469 has a much lower $\mathrm{Km}_{\text {(DHA- ATP) }}$ ) with ScDAK1 definitely detoxified the DHA that may

470 accumulate by the action of the introduced OpGDH and CuFPS1 in this study. Deletion

471 of DAKs resulted in the accumulation of significant amounts of DHA during glucose

472 fermentation (Nguyen \& Nevoigt, 2009). In addition to detoxification, overexpressing

473 DAKs was thought to speed up the transfer of DHA to DHAP, where overexpressed

474 DAK1 reduces DHA accumulation by nearly $16 \%$ compared with that of the

475 overexpressed strain $O p G D H$ (Klein et al, 2016). Although we tested the extracellular

476 accumulation of both DHA and DHAP in our recombinant strains, we found that their

477 concentrations were below detectable levels. This may be because our ancestor strains

478 have DAKs activity at $3.9 \mu \mathrm{mole} / \mathrm{min} / \mathrm{mg}$ of cell extract protein (Table 2). Nevertheless,

479 with the genetic modifications presented during the introduction of CuFPS1 and OpGDH,

480 while overexpressing ScDAK1 and ScDAK2, DHAP may have intracellularly

481 concentrations of influxes into the glycerol biosynthesis and G3P-pathways through

482 ScGPD2 or to saturate the native activity of ScTPI1 to convert into the pentose phosphate

483 pathway, especially with the presence of glucose (Grüning et al, 2014). Through scrutiny

484 of the previous studies in which the activity of SCTPI1 was abolished, we recognised the

485 pivotal role of overexpressing ScTPI1 in the current study, where the intracellular

486 concentration of DHAP accumulated to 30-fold concentrations (Shi et al, 2005) and when

487 this deactivation was further coupled with additional deletions of ScNDE1, ScNDE2 and 
ScGUT2, the fermentation product shifted from ethanol to glycerol (Overkamp et al, 2002).

However, integrating one copy of the whole DHA pathway with LINoxE generated the ability in yeast to convert all supplemented glucose and glycerol to ethanol. Nonetheless, we recognised that the conversion efficiency may still be affected by the robustness of native programmed glycolysis. Besides, the availability of ATP for DAKs was proposed as another limiting factor because DHA accumulation was decreased by nearly 58\% with replacing a phosphorylation pathway through GUT1 by a synthetic DHA pathway (Klein et al, 2016). Hence, this obstacle could be overcome by further strengthening of the entirety of the genes in the DHA pathway via additional copy under different expression systems with the replacement of GUT1. Potentially employing the strategy of multi-copy gene integrations while optimising the stoichiometries of the metabolic pathway considerably boosted production, e.g., six copies of the farnesene synthase gene, which was integrated into yeast to improve the synthesis of farnesene (Meadows et al, 2016). Here, with integration of a second copy of genes CuFBS1, OpGDH, ScDAK1 and ScTPI1, we further selected highly constitutive expressing systems in yeast (Ito et al, 2013; Yamanishi et al, 2013; Ito et al, 2016; Wei et al, 2017; Nambu-Nishida et al, 2018) to extend production levels and efficiencies, using the TEF1 promoter-CYC1 terminator, TYS1 promoter-ATP15 terminator, TDH3 promoter-mutated d22DIT1 terminator, and FBA1 promoter-TDH3 terminator, respectively. The other copies of CuFPS1, OpGDH, ScDAK1 and ScTPI1, which replaced ScGUT1, strengthened the specific activities of enzymes OpGDH, ScDAKs, and ScTPI1 by further $56 \%, 256 \%$ and $13 \%$, respectively, compared with the GN-FDT strain. Also, the $\mathrm{NADH} / \mathrm{NAD}^{+}$ratio decreased to 0.11 (Table 2). Interestingly, these reinforced activities with the possibility of providing ATP from the deletion of the GUT1 to the DAKs boosted production rates from $0.85 \sim 1.28 \mathrm{gL}^{-1} \mathrm{~h}^{-1}$ and the conversion efficiency reached $98 \%$ of the theoretical ratio in the rich medium (Table 4 [A]). Furthermore, the strain overrode the glucose repression during glycerol fermentation with glucose and produced up to $8.6 \%$ of ethanol (Table 4 [D]). For the efficient SK-FGG strain generated and its introduced pathway, oxygen availability became the limiting 
518 factor. Surprisingly, fermentation rates doubled with the fine-tuning to $>2 \mathrm{gL}^{-1} \mathrm{~h}^{-1}$ (Table

$5194[\mathrm{E}])$ although the $\mathrm{CE}$ decreased to $82.8 \%$ of the theoretical conversion. carbon source supplemented in YNB medium (without supplementary amino acids), to

522 ethanol with production rates of 0.25 and $0.44 \mathrm{gL}^{-1} \mathrm{~h}^{-1}$ with efficiencies 0.42 and $0.39 \mathrm{~g}^{\mathrm{e}} / \mathrm{g}^{\mathrm{g}}$, respectively with micro and semi-aerobic conditions (Table 5). Also, finetuning the oxygen availability shows notable effects during our study by decreasing the flasks sizes where it boosted the conversion efficiency from $78 \%$ to $84 \%$. This result matches with the previous finding, which revealed an improvement in ethanol production from $0.165 \mathrm{~g}^{\mathrm{e}} / \mathrm{g}^{\mathrm{g}}$ to $0.344 \mathrm{~g}^{\mathrm{e}} / \mathrm{g}^{\mathrm{g}}$ by reducing the oxygen availability during fermentation of glycerol in a buffered synthetic medium (Aßkamp et al, 2019). Later, NDE genes have reported as the main shuttles for re-oxidising cytosolic NADH during the growth and conversion of glycerol after replacing GUT1 with a DHA-FPS pathway in best natural positive-growing strain on synthetic medium supplemented by glycerol (Swinnen et al, 2013; Aßkamp et al, 2019). As a result, the conversion rates and maximum titer productivities are bounding by the oxidising shuttles within the respiratory chain and the availability of the final electron acceptor (Aßkamp et al, 2019). In addition to the competition with cell biomass formation (Aßkamp et al, 2019). As a result, the ethanol productivity rate was limited between $0.11 \sim 0.18 \mathrm{~g} \mathrm{gL}^{-1} \mathrm{~h}^{-1}$, although higher cell biomass levels (Aßkamp et al, 2019). Moreover, the highest ethanol titer was $15.7 \mathrm{~g} / \mathrm{L}$. showed higher potentials in productivity rates with higher efficiencies. Even when using glycerol as a sole carbon source in an unbuffered minimal medium, our engineered strain SK-FGG showed higher production rates ranged from 0.25 to $0.5 \mathrm{gL}^{-1} \mathrm{~h}^{-1}$ with higher conversion efficiencies set between 0.42 to $0.39 \mathrm{~g}^{\mathrm{e}} / \mathrm{g}^{\mathrm{g}}$, respectively. It is evidenced that SK-FGG fermented glycerol without linking with the respiratory routes and its limitation or the competition with cells formation, where SK-FGG reached the maximum growth of 8.55 9 OD at $12 \mathrm{~h}$ and continued glycerol consumption and ethanol production until

547 considering the start point of the ancestor strain, which showed growthless on glycerol as 548 a sole carbon source (Fig.S4) until reached by growth levels to 8.55 or 9 OD in SK-FGG 
549 (Table 5). With the productivity rates in this study, re-cycling NADH by LINoxE during

550 replacing $S c G P D 1$, which concomitant abolishing glycerol biosynthesis, is an innovative

551 point for fermenting glycerol and supported the conversion to unprecedented production

552 rates and efficiencies. Besides, the other novelty of overexpressing ScTPI1 and ScDAK2

553 during glycerol or/and glucose fermentation.

554 The remarkable difference between YP and YNB media is the increased shift

555 from ethanol production to acetic acid accumulation of $2.88 \mathrm{~g}$ from $54.01 \mathrm{~g}$ of consumed

556 glycerol in semi-aerobic conditions. As a result, the total conversion of acetic acid and

557 ethanol reached $90 \%$ of the theoretical value (Table 5). Like YP, a fully aerobic condition

558 promoted the acceleration of fermentation with the same efficiency of ethanol formation.

559 The content of amino acids in the medium has a crucial role in cell growth (Roberts et al,

560 2020); furthermore, we observed de novo biosynthesis of $\mathrm{NAD}^{+}$from tryptophan through

561 the kynurenine pathway (Panozzo et al, 2002; Kato \& Lin, 2014). Nicotinic acid, 562 nicotinamide, quinolinic acid and nicotinamide riboside can salvage the $\mathrm{NAD}^{+}$ 563 biosynthesis (Kato \& Lin, 2014). In this regard, nicotinic acid is auxotrophic under

564 anaerobic conditions in S. cerevisiae (Panozzo et al, 2002). These limitations may explain

565 the slower conversions and lower efficiency of ethanol production from glycerol when 566 using YNB (without supplemented amino acids) compared when using YP medium. We 567 must emphasise that strain SK-FGG was unable to ferment glycerol under the strictly 568 anaerobic condition in our experiments, which used YNB medium (Table 5). 569 Undoubtedly, there are no shuttles for the renovation of NADH under anaerobic 570 conditions. This finding agrees with the previously published data on the effect of oxygen 571 limitation on preventing the production of DHA during the fermentation of glucose 572 (Nguyen \& Nevoigt, 2009). Therefore, this study shows that the recycling of cofactors is 573 sufficient for complementing the robust oxidation of the DHA pathway for efficient 574 utilisation of glycerol to produce bioethanol or other bio-based chemicals. We are 575 currently working to further engineer a glycerol fermentation pathway while decreasing 576 dependence on oxygen as well as increasing the efficiencies of fermentation and utilising 577 the high reduction merit of glycerol for improving the fermentation efficiencies of other 578 carbons, while looking forward use crude glycerol from biodiesel industries in the next 579 studies. 
In summary, we show here the efficient modelling of glycerol traffic to ethanol production in S. cerevisiae. This systematic metabolic engineering includes integration of the following: (i) imposing vigorous expression of all genes in the glycerol oxidation pathway DHA including TPI1; (ii) prevalence of glycerol oxidation by an oxygendependent dynamic by the water-forming NADH oxidase LINoxE, which controls the reaction stoichiometries with regeneration of the cofactor $\mathrm{NAD}^{+}$; (iii) revoking the first step of both glycerol biosynthesis and glycerol catabolism through G3P (Fig. 1). Our study provides an advanced example of metabolic engineering for re-routing glycerol traffic in S. cerevisiae while tracking ethanol production to levels that have not yet been attained within any other safe model organisms, either native or genetically engineered (Yu et al, 2010; Yazdani \& Gonzalez, 2008; Trinh \& Srienc, 2009; Loaces et al, 2016; Aßkamp et al, 2019). Enormous global demands for bioethanol have been reported despite limited resources. Thus, this restricts global annual bioethanol production to approximately 29.1 million gallons, which represents $<2.7 \%$ of transportation fuels (World bioenergy association, 2018; Renewable Fuels Association, 2018; Garside, 2020). Therefore, the present metabolic strategy represents a pivotal breakthrough in the field of utilising the glycerol surplus and provides promising scenarios for biorefinery using the glycell process. The outcome of this study promotes the association between bioethanol or bio-based chemicals and biodiesel industries, which may develop expansions without overburdening sustainability, and may also prevent a decrease in present glycerol prices as well as broaden the horizons of glycerol-producing chemicals and biofuels.

\section{Material and methods}

\section{Strains, primers, cassettes, and plasmids constructions.}

Ancestor and all recombinant strains used in this study are listed in Table 1 and Fig. S3. The plasmids used in this study are listed in Table 6. All primers used here are listed in Table S1. Details of DNA fragments, cassettes and plasmids construction are explained as follows:

Construction of pPGK-ScTPI1, ScDAK2, ScDAK1 ScGCY1, ScSTL1 and pPGKScTPI1, ScGUT2, ScGUT1, ScSTL1 plasmids. 
609 We obtained the genes from genomic DNA of the ancestor strain D452-2 to clone the

610 plasmids in this section. Initially, cell walls were disrupted by resuspension with picked

611 cells in $20 \mu \mathrm{l}$ of $30 \mathrm{mM} \mathrm{NaOH}$ at $95^{\circ} \mathrm{C}$ for $10 \mathrm{~min}$, and then used directly as a template

612 for PCR; $1 \mu 1$ of fresh disrupted cells are suitable for a $50 \mu 1$ of PCR mixture. All primers

613 used to obtain the native genes were designed based on the sequences available on the

614 Saccharomyces Genome Database (SGD): https://www.yeastgenome.org/. For

615 assembling the plasmids pPGK-ScTPI1, pPGK-ScDAK2, pPGK-ScDAK1, pPGK-

616 ScGCY1 and pPGK-ScSTL1, the following genes from genomic DNA of the ancestral

617 strain were obtained by PCR: STL1, GCY1, DAK1\&2 and TPI1. High fidelity

618 polymerisation of KOD-plus neo with their corresponded primers (Table S1, section 1)

619 was used during this amplification. The Xhol site of DAK2 was deleted before cloning.

620 Genes were purified from the PCR mixtures using columns and accessories obtained

621 from Nippon Genetics Co., Ltd., and their cohesive ends were formed according to the

622 designated primers and restriction enzymes. We first separately cloned each gene in the

623 pPGK/URA3 plasmid (Kang et al, 1990) under the control of the expression system PGK

624 promoter and its terminator (Table 6). We further replaced the URA3 gene in a pPGK-

625 URA3 plasmid with an HIS3 (Rose et al, 1991) gene using a synthetically added overlap

626 sequence from the pPGK plasmid to the HIS3 marker using PCR and primers (Table S1,

627 section 3). A Gibson Assembly Master Mix was then used to assemble the overlapping

628 ends of the two fragments to form the PGK-HIS3 plasmid. With construction of the

629 pPGK-HIS3 plasmid (Table 6), we selected the HIS3 locus for homologous

630 recombination in $S$. cerevisiae after linearising the plasmid at the BsiWI site. We

631 obtained the plasmids and confirmed their gene sequences via sequencing using relevant

632 primers (Table S1, section 2). Next, we cut the XhoI/SalI-TPI1 cassette and inserted it

633 into XhoI/SalI sites of a newly constructed pPGK-HIS3 plasmid. Then the genes and

634 their systems were integrated together into one plasmid by connecting the DAK2 set into

635 the SalI site of the template plasmid initiated here by pPGK-TPI1. The non-reopened

636 ligations (XhoI/SalI sites) were used repeatedly during the ligation of new cassettes to

637 form new plasmids. DAK1, GCY1 and STL1 were repeatedly combined. Ultimately, the

638 pPGK-ScTPI1-ScDAK2-ScDAK1-ScGCY1-ScSTL1 plasmid was constructed (Table 6). 
639 Continuing with the same procedures, the plasmid pPGK-ScTPI1-ScGUT2-ScGUT1-

640 ScSTL1 was also established.

641 Construction of TDH3p-d22DIT1t, TDH3-d22-OpGDH and TDH3-d22-LINoxE 642 plasmids.

643 Cassette 1: partial end of GPD1promoter-TDH3p-d22DIT1terminator-partial front 644 side of GPD1 terminator and TDH3p-d22DIT1t plasmid. The mutated terminator $645 \mathrm{~d} 22 \mathrm{DIT} 1 \mathrm{t}$ was purchased from Integrated DNA Technology (IDT; Tokyo, Japan) 646 according to the published sequences (Ito et al, 2016). TDH3 promoter magnified from 647 the genomic DNA of the ancestor strain D452-2 using PCR and the designated primers 648 (Table S1, Section 4). All primers were purchased from FASMAC Company, Japan. 649 Moreover, flanking sequences added upstream of the promoter and downstream of the 650 terminator using the feature of PCR polymerisation with primers possessing a desired 651 long tail, and a further extension to those flanking sequences with the addition of 652 restriction sites was accomplished by PCR in the second step (Table S1, section 4). Then, 653 cohesive ends of those coupled DNA fragments were processed by the restriction 654 enzymes XhoI, NotI for the first fragment and NotI, SalI for the second. After 655 purification of the fragments using agarose gels and columns (Nippon Genetics Co., 656 Ltd.), one-step cloning was employed coupled the TDH3 promoter and mutated DITI 657 terminator into XhoI/Sall of PGK/URA3 plasmid. Then the TDH3p-d22DIT1t- URA3 658 plasmid was constructed (Table 6).

659 Cassette 2: partial end of GPD1promoter-TDH3p-OpGDH-d22DIT1t-partial front 660 side of GPD1terminator and TDH3-d22-OpGDH plasmid. The previously constructed 661 TDH3p-d22DIT1t/URA3 plasmid was used as a template for construction of the next 662 plasmid by further cloning $O p G D H$, deposited in GenBank under the accession number 663 XP_018210953.1. Synthetic $O p G D H$ was purchased from IDT. Primers are listed in 664 Section 4 in Table S1, and full sequences are available in Table S2.

665 Cassette 3: partial end of GPD1promoter-TDH3p- LINoxE -d22DIT1t-partial front 666 side of GPD1 terminator and TDH3-d22- LINoxE plasmid. We also purchased the 667 water-forming NADH oxidase gene of Lactococcus lactis (IDT) based on sequence 
available on gene bank accession number AAK04489.1 and cloned it into TDH3pd22DIT1t to assemble TDH3-d22- LINoxE plasmid (Tables 6 and S2).

Cassette 4: partial end of GPD2promoter-TDH3p- LINoxE -d22DIT1t-partial front side of GPD2 terminator and TDH3-d22- LINoxE plasmid. In this step, we replaced the flanking sequences of the GPD1 promoter and terminator with GPD2 using PCR and the primers listed in Section 5 - of Table S1.

\section{Construction of multiplex pCAS-gRNA-CRISPR systems.}

The multiplex pCAS-gRNA system was a gift from Prof. Jamie Cate (Addgene plasmid \# 60847; https://www.addgene.org/60847/) (Ryan et al, 2014). We used an online tool for the rational design of CRISPR/Cas target to allocate the highest probability of the on-target sites for the gRNA in the genomic DNA of $S$. cerevisiae (https://crispr.dbcls.jp/) (Naito et al, 2015). Accordingly, the sequences of the primers were designed based on the previously allocated sequence (20 bp before the PAM), with another $20 \mathrm{bp}$ from sgRNA or HDV ribozyme for overlap (Table S1, sections 4.2, 5.1 and 7.1). First, PCR was used to synthesise two fragments from the template, the pCASgRNA plasmid. The first was amplified using the forward primer called pCas forward (For) located upstream of the gRNA scaffold at the SmaI site of pCas, plus the antisense primer with a reverse sequence of the target gRNA. The second fragment was amplified by forward primer with a sense sequence of gRNA and the reverse primer called pCas reverse (Rev) located downstream of the gRNA scaffold (Table S1, section 4.2). After purifying each DNA segment, overlapping and integration was carried out by PCR using the pCas For and Rev primers. The produced fragment was then restricted to the SmaIPstI sites for cloning into a truncated pCAS-gRNA plasmid with SmaI-PstI. As a result, a new multiplex pCAS-gRNA plasmid was formed. Steps have been repeatedly performed with constructing all multiplex pCAS-gRNA plasmids targeting ScGPD1, ScGPD2 and GUT1 (Table 6). We confirmed the newly constructed systems by sequencing their entire scaffolds.

Construction of pAUR101- CuFPS1 and pAUR101-CuFPS1,ScTPI1, ScDAK2, ScDAK1 plasmids. 
698 Center (NBRC) of National Institute of Technology and Evaluation (Japan) and was used as a template for obtaining the gene glycerol facilitator FPS1 (CuFPS1). The sequence of CUFPS1 was included in the deposited gene bank accession number BAEL01000108.1. The original pAUR101 plasmid was purchased from Takara Bio, Inc., Japan, and the primers were used to establish this plasmid listed (Table S1, section 6). A full sequence for the cassette PGK-CUFPS1-RPL41Bt was transferred (Table S2). First, we constructed a pAUR101-PGKp-RPL41Bt vector by one-step cloning of the SmaINot1 PGK promoter (fragment 1) and NotI-SalI-RPL41B terminator (fragment 2) into the SmaI-SalI pAUR101 vector and then cloning a cohesive ended NotI-CuFPS gene into the dephosphorylated NotI site of pAUR101-PGK-RPL41B vector to assemble pAUR101PGKp-CUFPS1-RPL41Bt vector. To constitute the pAUR101CUFPS1, ScTPI1, ScDAK2, ScDAK1 plasmid, we detached the set of cassettesScTPI1, ScDAK2 and ScDAK1 - from previously constructed plasmids, pPGKScTPI1, ScDAK2 and ScDAK1 (Table S1), using restriction enzymes Xhol-SalI and reinserted that set of cassettes (ScTPI1, ScDAK2 and ScDAK1) into the Sall site of the pAUR101-PGK-CuFPS1-RPL41B plasmid (Table 6).

\section{Construct Module M1; CuFPS1, OgGDH, ScDAK1, ScTPI1 cassettes with flanking} sequences of GUT1 promoter and terminator in plasmid pAUR101.

We first obtained all fragments which could form the module M1 separately by PCR (Fig. S2); the CuFPS1, OpGDH genes, and mutated d22DIT terminator amplified from their synthetic DNA stocks, whereas other fragments were magnified from the genomic DNA of the D452-2 strain (Fig. S2). The full sequence of the module M1 is also accessible (Table S2), and the primer details are listed in Table S1, Section 7. Purification of the 12 amplified DNA fragments was carried out on 1\%-2\% agarose gel and then recovered by the FastGene Gel/PCR Extraction Kit (Nippon Genetics Co. Ltd) according to the manufacturer's protocol. We accordingly obtained highly purified fragments before the onset of assembly using the Gibson Assembly Master Mix. We effectively joined the first three segments seamlessly, as well as for each of the next three fragments according to the manufacturer's protocol (Gibson). We also directly amplified each set by PCR and 
727 then purified these again on an agarose gel. We repeatedly gathered the first six

728 segments, as well as the other six fragments, and then assembled the whole module M1.

729 We further added the SacI site upstream of the module M1 and the SmaI site

730 downstream. These restriction sites were provided for cloning the module M1 into SacI-

731 SmaI sites of pAUR101 vector to form pAUR 101-M1 (Table 6). Finally, we transferred

732 the vector pAUR 101-M1 into E. coli as described previously and confirmed the accurate

733 structure of M1 by sequencing the whole module M1 from pAUR-M1.

\section{Transformation and recombination of strains in this study.}

All the previous plasmids stored in E. coli NEB 10-beta for further uses of production of the required plasmids or cassettes, using the heat shock method according to the procedures provided with the competent cells. All plasmid extractions were performed using the QIAprep Spin Miniprep Kit following the manufacturer's protocol. All measurements of DNA were estimated using BioSpec-nano (Shimadzu, Japan), and DNA was stored at $-20^{\circ} \mathrm{C}$ for future use. Yeast transformation by Fast Yeast Transformation $^{\mathrm{TM}}$ kit (Takara Bio) was used for integrated linear pAUR101 vector and its associated genes in the AUR1-C locus, as well as linear pPGK plasmid with its cloned genes in either HIS3 or URA3 loci (Hosaka et al, 1992). For achieving genome editing and the replacement of ScGPD1, ScGPD2 and GUT1 genes with its designated DNA repairing cassette or module, we used the protocol of CRISPR-Cas9 genome engineering in S. cerevisiae cells (Ryan et al, 2016). We confirmed target replacements using PCR for the inserted repairing cassettes with primers from upstream and downstream of the flanking recombined loci. The primers used are listed in (Table S1). Furthermore, we cultivated up to 10 generations of the selected evolved strains to confirm the loss of pCAS plasmid and re-confirm the recombination. All recombination strains and their genotypes are listed in Table 1.

\section{Preparation of cell-free extract.}

Cell proteins were extracted as previously described (Nguyen \& Nevoigt, 2009; Khattab et al, 2013) with some modifications. The recombinant strains listed in Table 2 were cultivated in closed 50-ml Falcon tubes with $10 \mathrm{ml}$ of YP medium supplemented 
with $(\mathrm{w} / \mathrm{v}) 1.5 \%$ glucose and $7 \%$ glycerol $\left(\mathrm{YPD}_{15} \mathrm{G}_{70}\right)$ for $15 \mathrm{~h}$ with $200 \mathrm{rpm}$ of shaking. Cell pellets were harvested by centrifugation at $4000 \mathrm{rpm}$ for $2 \mathrm{~min}$ at $4{ }^{\circ} \mathrm{C}$, then washed with $20 \mathrm{ml}$ of $100 \mathrm{mM}$ of HEPES buffer ( $\mathrm{pH} \mathrm{7.4)}$ and centrifuged again. Then, the cell pellets were lysed in $1 \mathrm{ml}$ of HEPES buffer supplemented with $1 \mathrm{mM} \mathrm{MgCl}$ and $10 \mathrm{mM}$ 2-mercaptoethanol with approximately $400 \mathrm{mg}$ of glass beads in a 2-ml Eppendorf tube. The lysis was accomplished by vigorous shaking by bench vortex with six-time intervals on ice for 30 seconds. The crude proteins were separated from the glass beads and cell debris by two rounds of centrifugation at $15000 \mathrm{rpm}$ at $4^{\circ} \mathrm{C}$ for $5 \mathrm{~min}$. Total protein concentration was estimated using Bio-RAD Quick Start Bradford $1 \mathrm{x}$ dye reagent by measuring the absorbance at 595nm using BSA as a standard.

\section{Enzyme assays.}

The specific activity of glycerol dehydrogenase (GDH) was assayed by monitoring the increase of NADH absorbance at $340 \mathrm{~nm}$ within $1 \mathrm{~min}$ (UV-2700; UVVIS spectrophotometer, Shimadzu, Japan) in a 1-ml mixture of $80 \mathrm{mM}$ HEPES buffer (pH 7.4), $5 \mathrm{mM} \mathrm{NAD}{ }^{+}, 100 \mathrm{mM}$ glycerol and $10 \mu 1$ of crude extract. The activity of TPI1 was assayed as described previously (Plaut \& Knowles, 1972) with some modifications. The reaction mixture was $1 \mathrm{ml}$ in volume and was composed of $100 \mathrm{mM}$ triethanolamine hydrochloride ( $\mathrm{pH} 7.53), 2.5 \mathrm{mM} \mathrm{NAD}^{+}, 10 \mathrm{mM}$ DHAP and $10 \mu \mathrm{l}$ of crude extract. Dihydroxyacetone kinase was assayed using a universal kinase activity kit according to the manufacturer's instructions (Catalog Number EA004).

\section{Intracellular concentration of $\mathrm{NAD}^{+} / \mathrm{NADH}$.}

The cellular contents of $\mathrm{NAD}^{+} / \mathrm{NADH}$ were colorimetric quantitatively determined at $565 \mathrm{~nm}$ using a BioAssay Systems (E2ND-100) EnzyChrom ${ }^{\mathrm{TM}} \mathrm{NAD}^{+}$ /NADH Assay Kit.

\section{Fermentation procedures and analysis.}

The initial fermentation experiments were performed in 100-ml Erlenmeyer flasks with $20 \mathrm{ml}$ of culture medium for the estimation of micro-aerobic conditions and 20:200 liquid: flask volume for the semi-aerobic case. For the aerobic conditions, $20 \mathrm{ml}$ of culture medium injected into 300 or 500-ml flasks respectively, with YNB and YP medium. 
785 Three different agitation speeds were tested through this study (150, 180 and $200 \mathrm{rpm})$ at

$78630^{\circ} \mathrm{C}$, which stated in the legends of each table or figure. For strict anaerobic condition,

$78720 \mathrm{ml}$ of YNB medium was transferred into sterilised $50-\mathrm{ml}$ vial and then caped with

788 precision seal septa cap. Afterward, the nitrogen gas was purged into the culture medium

789 through one use of Terumo needles to replace the dissolved oxygen and the air. Sampling

790 was also drawn through new Terumo needles. The fermenter cells were harvested from

791 the same volume of the pre-culture YPD medium for approximately $15 \mathrm{~h}$. For SK-FGG,

792 the cultivation culture medium was YPD20G70. Cells were harvested by centrifugation at

$7936000 \times \mathrm{g}$ for $5 \mathrm{~min}$ at $4^{\circ} \mathrm{C}$ and washed with sterile water. Then, collected cells were re-

794 supplemented with the YP medium with glucose, glycerol, or both, as shown in Figures

795 2-3 and tables 2-5. Different initial concentrations were used to determine the

796 fermentation abilities at those concentrations as well as with the fed-batch to estimate the

797 maximum product under these unprecedented fermentation conditions. The cell density

798 was monitored using spectrophotometry at $600 \mathrm{~nm}$ (AS ONE, Japan).

799 All analyses were performed using auto-sampling a 10 microliter to injected in an

800 Aminex HPX-87H column (Bio-Rad Laboratories, Hercules, CA, USA), analysed in a

801 refractive index detector (RID-10A; Shimadzu), and a prominence diode array detector

802 (SPD-M20A; Shimadzu) equipped with an auto-sampling ultra-fast liquid

803 chromatography (Shimadzu, Japan). Fractionation was accomplished at a flow rate of 0.6

$804 \mathrm{ml} / \mathrm{min}$ with $5 \mathrm{mM} \mathrm{H}_{2} \mathrm{SO}_{4}$ as the mobile phase at $50^{\circ} \mathrm{C}$. Reactant concentrations were

805 estimated by monitoring the peak areas compared with the standards of the authenticating

806 reactant's glucose, glycerol, ethanol, acetic acid, pyruvate, succinic acid and

807 acetaldehyde, which detected by RID. We used the SPD to estimate DHAP and DHA.

808 DHA was monitored by the RID where the glycerol peaks overlapped. The measurements

809 of DHAP were more precise when using the SPD compared when using the RID, and

810 there was partially overlapping of the frequently negative peak of the difference of

811 samples with the mobile phase. The detectable quantities were reported in the tables or

812 figures and the quantities under the undetectable levels were omitted.

\section{ACKNOWLEDGMENTS}


814 Funding: This work was supported by Mission 5-2 Research Grant from the Research

815 Institute for Sustainable Humanosphere, Kyoto University.

Author contributions: S.M.R.K. was responsible for the research idea, conception, planning and organisation of the experiments. S.M.R.K. also provided the information for purchasing the strains, chemicals, and toolboxes for the genetic engineering, performed the experiments and analysed and discussed the results. S.M.R.K. wrote, revised, and submitted the manuscript. T.W. was responsible for all financial support and provided all chemicals and equipment. T.W. was involved in the research idea, the conception, the planning and organisation, the discussion of the results and the manuscript revision and submission.

\section{Competing interests:}

The authors state that there are no competing interests to declare.

\section{Data and materials availability:}

All necessary data required to assess our findings are available in this manuscript or its supplementary data. Further details related to this article may be requested from the authors.

\section{References}

Ansell R, Granath K, Hohmann S, Thevelein JM, Adler L (1997) The two isoenzymes for yeast $\mathrm{NAD}^{+}$-dependent glycerol 3-phosphate dehydrogenase encoded by GPD1 and GPD2 have distinct roles in osmoadaptation and redox regulation. EMBO J 16(9): $2179-218$

Aßkamp MR, Klein M, Nevoigt E (2019) Involvement of the external mitochondrial NADH dehydrogenase Nde1 in glycerol metabolism by wild-Type and engineered Saccharomyces cerevisiae strains. FEMS Yeast Res 1: 19(3)

Aßkamp MR, Klein M, Nevoigt E (2019) Saccharomyces cerevisiae exhibiting a modified route for uptake and catabolism of glycerol forms significant amounts of 
ethanol from this carbon source considered as 'non fermentable'. Biotechnol Biofuels 12: 257

Babazadeh R, Lahtvee PJ, Adiels CB, Goksör M, Nielsen JB, Hohmann S (2017) The yeast osmostress response is carbon source dependent. Sci Rep 7: 990

Bae S-J, Kim S, Hahn J-S (2016) Efficient production of acetoin in Saccharomyces cerevisiae by disruption of 2,3-butanediol dehydrogenase and expression of NADH oxidase. Sci Rep 6: 27667

Deepak D, Gregory S (2011) Relative potential of biosynthetic pathways for biofuels and bio-based products. Nat Biotechnol 29: 1074-1078

Gancedo C, Gancedo JM, Sols A (1968) Glycerol metabolism in yeasts pathways of utilization and production. Eur J Biochem 5: 165-172

Garside M (2020) STATISTA. Fuel ethanol production worldwide in 2019, by country. https://www.statista.com/statistics/281606/ethanol-production-in-selected$\underline{\text { countries/ }}$

Grauslund M, Lopes JM, Ronnow B (1999) Expression of GUT1, which encodes glycerol kinase in Saccharomyces cerevisiae, is controlled by the positive regulators Adr1p, Ino2p and Ino4p and the negative regulator Opilp in a carbon source-dependent fashion. Nucleic Acids Res 27: 4391-4398

Grauslund M, Ronnow B (2000) Carbon source-dependent transcriptional regulation of the mitochondrial glycerol-3-phosphate dehydrogenase gene, GUT2, from Saccharomyces cerevisiae. Can J Microbiol 46: 1096-1100

Grüning NM, Du D, Keller MA, Luisi BF, Ralser M (2014) Inhibition of triosephosphate isomerase by phosphoenolpyruvate in the feedback-regulation of glycolysis. Open Biol 4: 130232 
Ho P-W, Swinnen S, Duitama J, Nevoigt E (2017) The sole introduction of two single point mutations establishes glycerol utilization in Saccharomyces cerevisiae CEN.PK derivatives. Biotechnol Biofuels 10: 10

Hosaka K, Murakami T, Kodaki T, Nikawa J, Yamashita S (1990) Repression of choline kinase by inositol and choline in Saccharomyces cerevisiae. J Bacteriol 172(4): 2005-12

Hosaka K, Nikawa J, Kodaki T, Yamashita S (1992) A dominant mutation that alters the regulation of INO1 expression in Saccharomyces cerevisiae. J Biochem 111: 352358

Islam Z-U, Klein M, Ødum ASR, Nevoigt E (2017) A modular metabolic engineering approach for the production of 1,2-propanediol from glycerol by Saccharomyces cerevisiae. Metab Eng 44: 223-35

Ito $\mathrm{Y}$, Kitagawa $\mathrm{T}$, Yamanishi M, Katahira S, Izawa S, Irie K, Furutani-Seiki M, Matsuyama T (2016) Enhancement of protein production via the strong DIT1 terminator and two RNA-binding proteins in Saccharomyces cerevisiae, Sci. Rep 6: 36997

Ito Y, Yamanishi M, Ikeuchi A, Imamura C, Tokuhiro K, Kitagawa T, Matsuyama T (2013) Characterization of five terminator regions that increase the protein yield of a transgene in Saccharomyces cerevisiae. J Biotechnol 168: 486-492

Kampf G, Todt D, Pfaender S, Steinmann E (2020) Persistence of coronaviruses on inanimate surfaces and their inactivation with biocidal agents. J Hosp Infect 104: $246-251$

Kang YS, Kane J, Kurjan J, Stadel JM, Tipper DJ (1990) Effects of expression of mammalian $G$ alpha and hybrid mammalian-yeast $G$ alpha proteins on the yeast pheromone response signal transduction pathway. Mol Cell Biol 10: 2582-2590 
Kato M, Lin SJ (2014) Regulation of $\mathrm{NAD}^{+}$metabolism, signaling and compartmentalization in the yeast Saccharomyces cerevisiae. DNA Repair 23: 4958

Khattab SMR, Kodaki T (2014) Efficient bioethanol production by overexpression of endogenous Saccharomyces cerevisiae xylulokinase and NADPH-dependent aldose reductase with mutated strictly $\mathrm{NADP}^{+}$-dependent Pichia stipitis xylitol dehydrogenase. Process Biochem 49: 1838-1842

Khattab SMR, Saimura M, Kodaki T (2013) Boost in bioethanol production using recombinant Saccharomyces cerevisiae with mutated strictly NADPH-dependent xylose reductase and NADP+-dependent xylitol dehydrogenase. J Biotechnol 165: 153-156

Khattab SMR, Watanabe T (2019) Bioethanol from sugarcane bagasse: Status and perspectives. In Bioethanol Production from Food Crops: Sustainable Sources, Interventions, and Challenges, Ramesh CR, Ramachandran S (eds) pp 187-212. Elsevier

Kim J-W, Lee Y-G, Kim S-J, Jin, Y-S, Seo, J-H. (2019) Deletion of glycerol-3-phosphate dehydrogenase genes improved 2,3-butanediol production by reducing glycerol production in pyruvate decarboxylase-deficient Saccharomyces cerevisiae. $J$ Biotechnol 304: 31-37

Kim J-W, Seo S-O, Zhang G-C, Jin Y-S, Seo J-H (2015) Expression of Lactococcus lactis NADH oxidase increases 2,3-butanediol production in Pdc-deficient Saccharomyces cerevisiae. Bioresour Technol 191: 512-519

Kim S, Hahn J-S. (2015) Efficient production of 2,3-butanediol in Saccharomyces cerevisiae by eliminating ethanol and glycerol production and redox rebalancing. Metab Eng 31: 94-101 
914 Klein M, Carrillo M, Xiberras J, Islam Z-U, Swinnen S, Nevoigt E (2016) Towards the exploitation of glycerol's high reducing power in Saccharomyces cerevisiae-based bioprocesses. Metab Eng 38: 464-472

Klein M, Islam Z-U, Knudsen PB, Carrillo M, Swinnen S, Workman M, Nevoigt E (2016) The expression of glycerol facilitators from various yeast species improves growth on glycerol of Saccharomyces cerevisiae. Metab Eng Commun 3: 252-257

Kodaki T, Yamashita S (1989) Characterization of the methyltransferases in the yeast phosphatidylethanolamine methylation pathway by selective gene disruption. Eur $J$ Biochcm 185: 243-251

Larsson C, Påhlman IL, Ansell R, Rigoulet M, Adler L, Gustafsson L (1998) The importance of the glycerol 3-phosphate shuttle during aerobic growth of Saccharomyces cerevisiae. Yeast 15;14(4): 347-357

Loaces I, Rodríguez C, Amarelle V, Fabiano E, Noya F (2016) Improved glycerol to ethanol conversion by E. coli using a metagenomic fragment isolated from an anaerobic reactor. J Ind Microbiol Biotechnol 43: 1405-1416

Lovins, AB, Datta EK, Bustnes O-E, Koomey JG \& Glasgow NJ (2004) Winning the Oil Endgame. Innovation for Profits, Jobs, and Security. Snowmass, CO: Rocky Mountain Institute, United States

Luque R, Herrero-Davila L, Campelo JM, Clark JH, Hidalgo JM, Luna D, Marinas JM, Romero AA (2008) Biofuels: a technological perspective. Energy Environ Sci 1: $542-564$

Lutstorf U, Megnet R (1968) Multiple forms of alcohol dehydrogenase in Saccharomyces cerevisiae. physiological control of ADH-2 and properties of ADH-2 and ADH-4. Arch Biochem Biophys 126: 933-944 
Meadows AL, Hawkins KM, Tsegaye Y, Antipov E, Kim Y, Raetz L, Dahl RH, Tai A, Mahatdejkul-Meadows T, Xu L et al (2016) Rewriting yeast central carbon metabolism for industrial isoprenoid production. Nature 537: 694-697

Molin M, Norbeck J, Blomberg A (2003) Dihydroxyacetone kinases in Saccharomyces cerevisiae are involved in detoxification of dihydroxyacetone. J Biol Chem 278(3): $1415-1423$

Murata K, Fukuda Y, Watanabe K, Saikusa T, Shimosaka M, Kimura A (1985) Characterization of methylglyoxal synthase in Saccharomyces cerevisiae. Biochem Biophys Res Commun 131: 190-198

Naito Y, Hino K, Bono H, Ui-Tei K (2015) CRISPRdirect: software for designing CRISPR/Cas guide RNA with reduced off-target sites. J Bioinform 31: 1120-1123

Nambu-Nishida Y, Sakihama Y, Ishii J, Hasunuma T, Kondo A (2018) Selection of yeast Saccharomyces cerevisiae promoters available for xylose cultivation and fermentation. J Biosci Bioeng 125(1): 76-86

Nguyen HT, Nevoigt E (2009) Engineering of Saccharomyces cerevisiae for the production of dihydroxyacetone (DHA) from sugars: A proof of concept. Metab Eng 11: 335-346

Nomanbhay S, Hussein R, Ong MY (2018) Sustainability of biodiesel production in Malaysia by production of bio-oil from crude glycerol using microwave pyrolysis: a review. Green Chem Lett Rev 11: 135-157

Ochoa-Estopier A, Lesage J, Gorret N, Guillouet SE (2011) Kinetic analysis of a Saccharomyces cerevisiae strain adapted for improved growth on glycerol: implications for the development of yeast bioprocesses on glycerol. Bioresour Technol 102(2): 1521-7

Ohashi Y, Watanabe T (2018) Catalytic performance of food Additives Alum, flocculating agent, $\mathrm{Al}(\mathrm{SO} 4) 3, \mathrm{AlCl} 3$ and other Lewis acids in microwave solvolysis 
of hardwoods and recalcitrant softwood for biorefinery. ACS Omega 3:1627116280

Overkamp KM, Bakker BM, Kötter P, Luttik MAH, van Dijken JP, Pronk, JT (2002) Metabolic Engineering of glycerol production in Saccharomyces cerevisiae. Appl Environ Microbiol 68(6): 2814-2821

Panozzo C, Nawara M, Suski C, Kucharczyka R, Skoneczny M, Bécam A M, Rytka J, Herbert, CJ (2002) Aerobic and anaerobic NAD ${ }^{+}$metabolism in Saccharomyces cerevisiae. FEBS Lett 517(1-3): 97-102

Peris D, Moriarty RV, Alexander WG, Baker EC, Sylvester K, Sardi M, Langdon QK, Libkind D, Wang Q-M, Bai F-Y et al. (2017) Hybridization and adaptive evolution of diverse Saccharomyces species for cellulosic biofuel production. Biotechnol Biofuels 10: 78

Plaut B, Knowles J R (1972) pH-dependence of the triose phosphate isomerase reaction. Biochem J 129(2): 311-320

Ragauskas AJ, Williams CK, Davison BH, Britovsek G, Cairney J, Eckert CA, FrederickJr WJ, Hallett JP, Leak DJ, et al (2006) The path forward for biofuels and biomaterials. Science 311: 484-489

Renewable Fuels Association. World Fuel Ethanol Production 2018 -. https://ethanolrfa.org/statistics/annual-ethanol-production/

Roberts GG, Hudson AP (2009) Rsflp is required for an efficient metabolic shift from fermentative to glycerol-based respiratory growth in S. cerevisiae. Yeast 26: 95-110

Roberts TM, Kaltenbach H-M, Rudolf F (2020) Development and optimisation of a defined high cell density yeast medium. Yeast 37(5-6): 336-347

Rose MD, Broach JR (1991) Cloning genes by complementation in yeast. Methods Enzymol 194: 195-230 
Ryan OW, Poddar S, Cate JHD. (2016) CRISPR-Cas9 Genome engineering in Saccharomyces cerevisiae cells. Cold Spring Harbor Protocol

Ryan OW, Skerker JM, Maurer MJ, Li X, Tsai CJ, Poddar S, Lee ME, DeLoache W, Dueber JE, Arkin AP, Cate JHD (2014) Selection of chromosomal DNA libraries using a multiplex CRISPR system. eLife 3: e03703

Saito H, Posas F (2012) Response to Hyperosmotic Stress. Genetics 192: 289-318

Semkiv M, Kata I, Ternavska O, Sibirny W, Dmytruk K, Sibirny A (2019) Overexpression of the genes of glycerol catabolism and glycerol facilitator improves glycerol conversion to ethanol in the methylotrophic thermotolerant yeast Ogataea polymorpha. Yeast 36: 329-339

Shi Y, Vaden DL, Ju S, Ding D, Geiger JH, Greenberg ML (2005) Genetic perturbation of glycolysis results in inhibition of de novo inositol biosynthesis. J Biol Chem 280(51): 41805-41810

Sprague GF, Cronan JE (1977) Isolation and characterization of Saccharomyces cerevisiae mutants defective in glycerol catabolism. J Bacteriol 129(3):1335-42

Swinnen S, Ho P-W, Klein M, Nevoigt E (2016) Genetic determinants for enhanced glycerol growth of Saccharomyces cerevisiae. Metab Eng 36: 68-79

Swinnen S, Klein M, Carrillo M, McInnes J, Nguyen HT, Nevoigt E (2013) Reevaluation of glycerol utilization in Saccharomyces cerevisiae: characterization of an isolate that grows on glycerol without supporting supplements. Biotechnol Biofuels 6: 157

Trinh CT, Srienc F (2009) Metabolic engineering of Escherichia coli for efficient conversion of glycerol to ethanol. Appl Environ Microbiol 75: 6696-6705

Turcotte B, Liang XB, Robert F, Soontorngun N (2010) Transcriptional regulation of nonfermentable carbon utilization in budding yeast. FEMS Yeast Res 10: 2-13 
1014 Vemuri GN, Eiteman MA, McEwen JE, Olsson L, Nielsen J (2007) Increasing NADH

1015

1016

1017

1018

1019

1020

1021

1022

1023

1024

1025

1026

1027

1028

1029

1030

1031

1032

1033

1034

1035

1036

1037

1038

oxidation reduces overflow metabolism in Saccharomyces cerevisiae. Proc Natl Acad Sci 104: 2402-2407

Wei L, Wang Z, Zhang G, Ye B (2017) Characterization of terminators in saccharomyces cerevisiae and an exploration of factors affecting their strength. Chem Bio Chem 18: $2422-2427$

Wei N, Quarterman J, Kim SR, Cate JH. Jin YS (2013) Enhanced biofuel production through coupled acetic acid and xylose consumption by engineered yeast. Nat Commun 4: 2580 .

World bioenergy association. WBA Global Bioenergy Statistics 2018 https://worldbioenergy.org/uploads/181203\%20WBA\%20GBS\%202018 hq.pdf

Xiberras J, Klein M, Nevoigt E (2019) Glycerol as a substrate for Saccharomyces cerevisiae based bioprocesses - Knowledge gaps regarding the central carbon catabolism of this 'non-fermentable' carbon source. Biotechnol Adv 37: 107378

Yamada-Onodera K, Yamamoto H, Emoto E, Kawahara N, Tani Y (2002) Charaterisation of glycerol dehydrogenase from a methylotrophic yeast Hansenula polymorpha DL-1, and its gene cloning. Acta Biotechnol 22: 337-353

Yamanishi M, Ito Y, Kintaka R, Imamura C, Katahira S, Ikeuchi A, Moriya H, Matsuyama T (2013) A genome-wide activity assessment of terminator regions in Saccharomyces cerevisiae provides a "terminatome" toolbox. ACS Synth Biol 2: $337-347$

Yazdani SS, Gonzalez R (2007) Anaerobic fermentation of glycerol: a path to economic viability for the biofuels industry. Curr Opin Biotechnol 18: 213-219

Yazdani SS, Gonzalez R (2008). Engineering Escherichia coli for the efficient conversion of glycerol to ethanol and co-products. Metab Eng 10: 340-351 
1039 Yu KO, Kim SW, Han SO (2010) Engineering of glycerol utilization pathway for ethanol 1040 production by Saccharomyces cerevisiae. Bioresour Technol 101: 4157-4161

1041

1042

1043

1044

1045

1046

1047

1048

1049

1050

1051

1052

1053
Zheng Z, Zou J (2001) The initial step of the glycerolipid pathway: identification of glycerol 3-phosphate/dihydroxyacetone phosphate dual substrate acyltransferases in Saccharomyces cerevisiae. J Biol Chem 276: 41710-41716

\section{Figures}

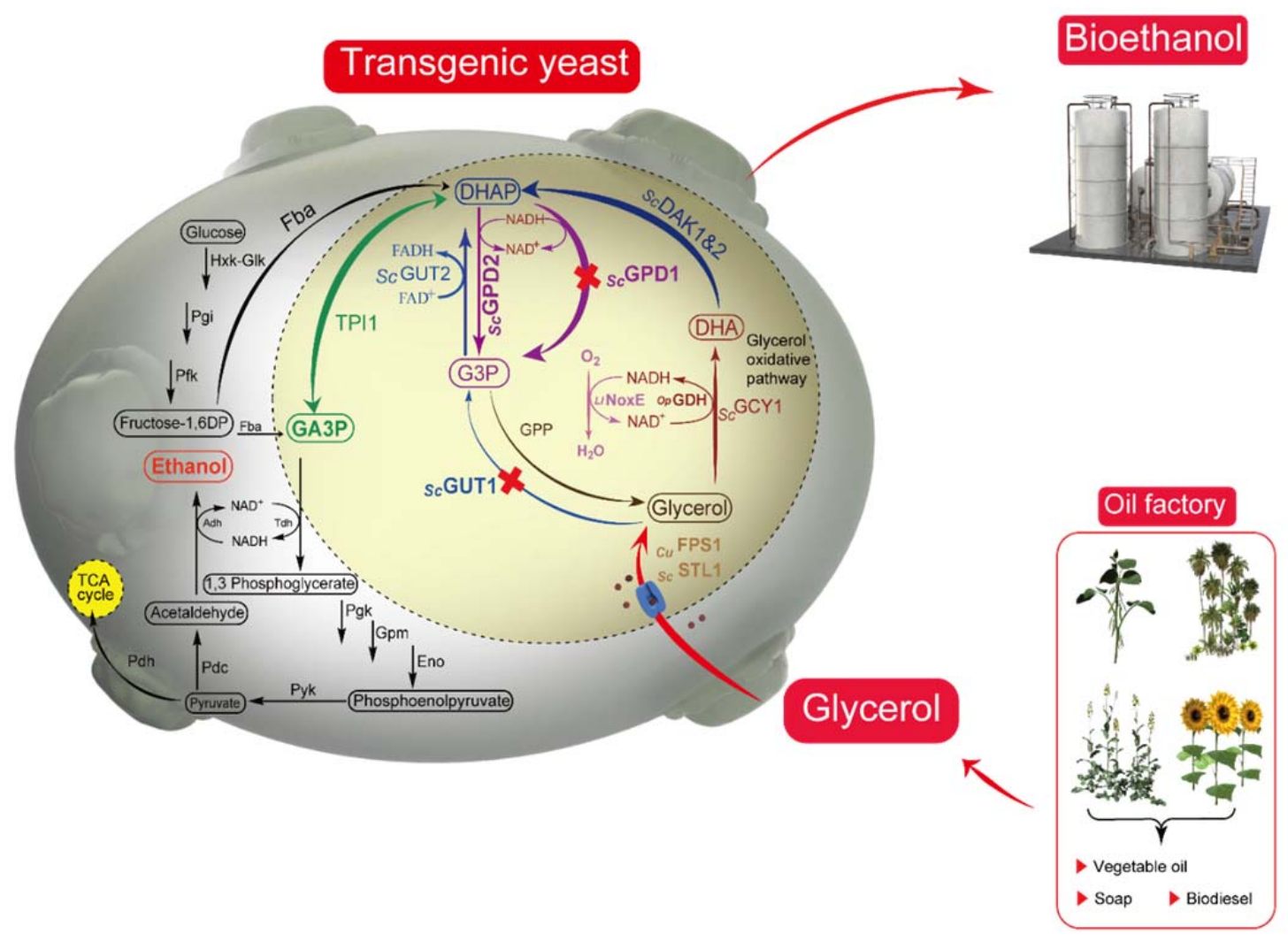

Fig. 1. Schematic diagram showing the integrative scenario of a biorefinery with new generation of glycerol fermenting yeast and redirection of glycerol influxes to ethanol production in Saccharomyces cerevisiae via retrofitted native glycerol anabolic and catabolic pathways using the robust oxidative route with renovation of the $\mathrm{NAD}^{+}$ cofactor via $\mathrm{O}_{2}$-dependent dynamics of water-forming $\mathrm{NADH}$ oxidase. During pathway re-routing, glycerol-3-phosphate dehydrogenase 1 (ScGPD1) and glycerol kinase 1 (GUT1) were knocked-out. A highlighted circle indicates the overexpressed indigenous S. cerevisiae enzymes dihydroxyacetone kinase (ScDAK) 1 and 2 as well as 
1054 triosephosphate isomerase (ScTPI) 1, heterologous glycerol dehydrogenase from 1055 Ogataea polymorpha $(\mathrm{Op} G D H)$, glycerol facilitator from Candida utilis (CuFPS1) and 1056 water-forming NADH oxidase from Lactococcus lactis subsp. lactis I11403 (LINoxE).

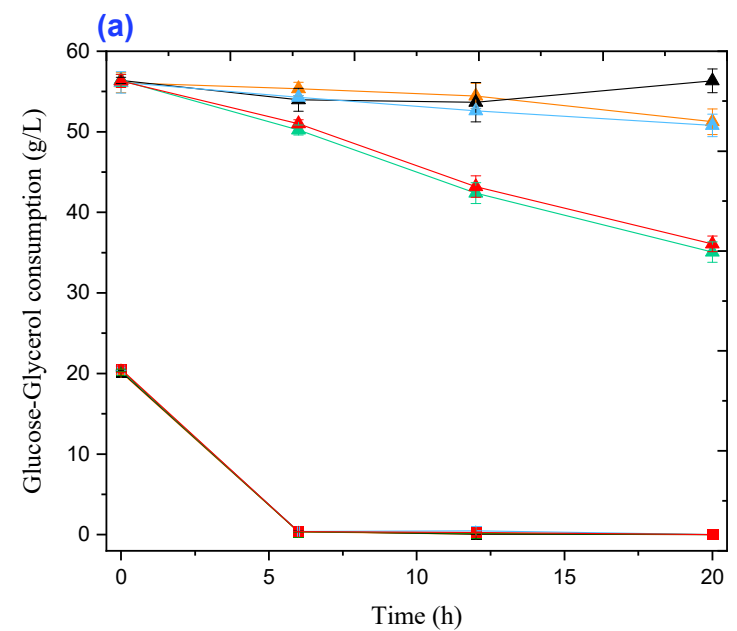

1057

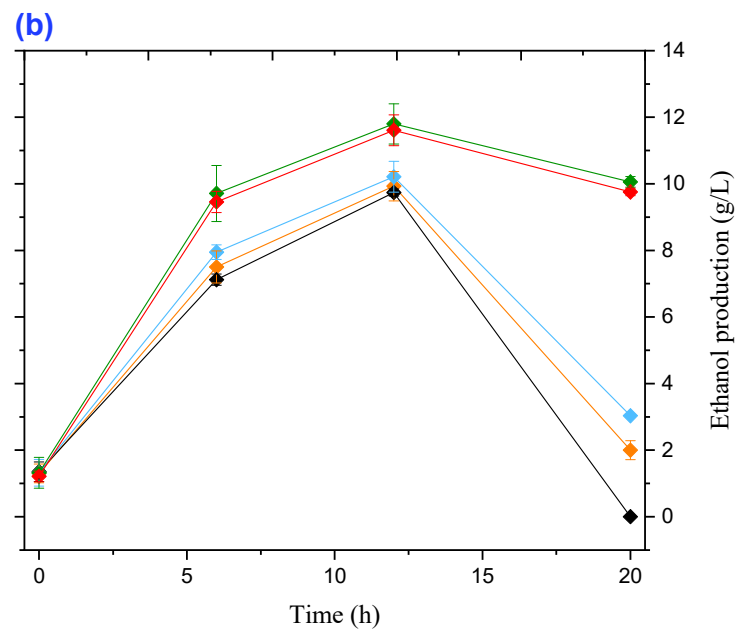

1058

1059 Fig. 2. Time course for fermentation of glucose-glycerol by S. cerevisiae in micro1060 aerobic condition (20:100ml; liquid medium: flask volume) at $30^{\circ} \mathrm{C}$ with shaking at 180 1061 rpm: ancestral strain (black lines); GF2 strain overexpressing endogenous oxidative 1062 (DHA) pathways ScSTL1, ScGCY1, ScDAK1, ScDAK2 and ScTPI1 (blue lines); GA2 1063 strain overexpressing endogenous phosphorylation (G3P) pathways ScSTL1, ScGUT1, 1064 ScGUT2 and ScTPI1 (orange lines); GDH strain overexpressing OpGDH (green lines); $1065 \Delta$ GUT1+ GDH (red lines); (a) glucose consumption (squares), glycerol consumption 1066 (triangles); (b) ethanol production (rhomboid symbols). Data are obtained from the 
1067 mean of three independent experiments run simultaneously to decrease time 1068 differences in sampling. Error bars represent the standard deviation of the mean $(\mathrm{n}=$ 1069 3). YP medium supplemented with (w/v) 2\% glucose, and 5.5\% glycerol was used.

1070 The only significant difference in growth rates at $\mathrm{OD}_{600}$ between the above strains was

1071 found on YNB medium and showed in the supplementary data (Fig. S4).

1072
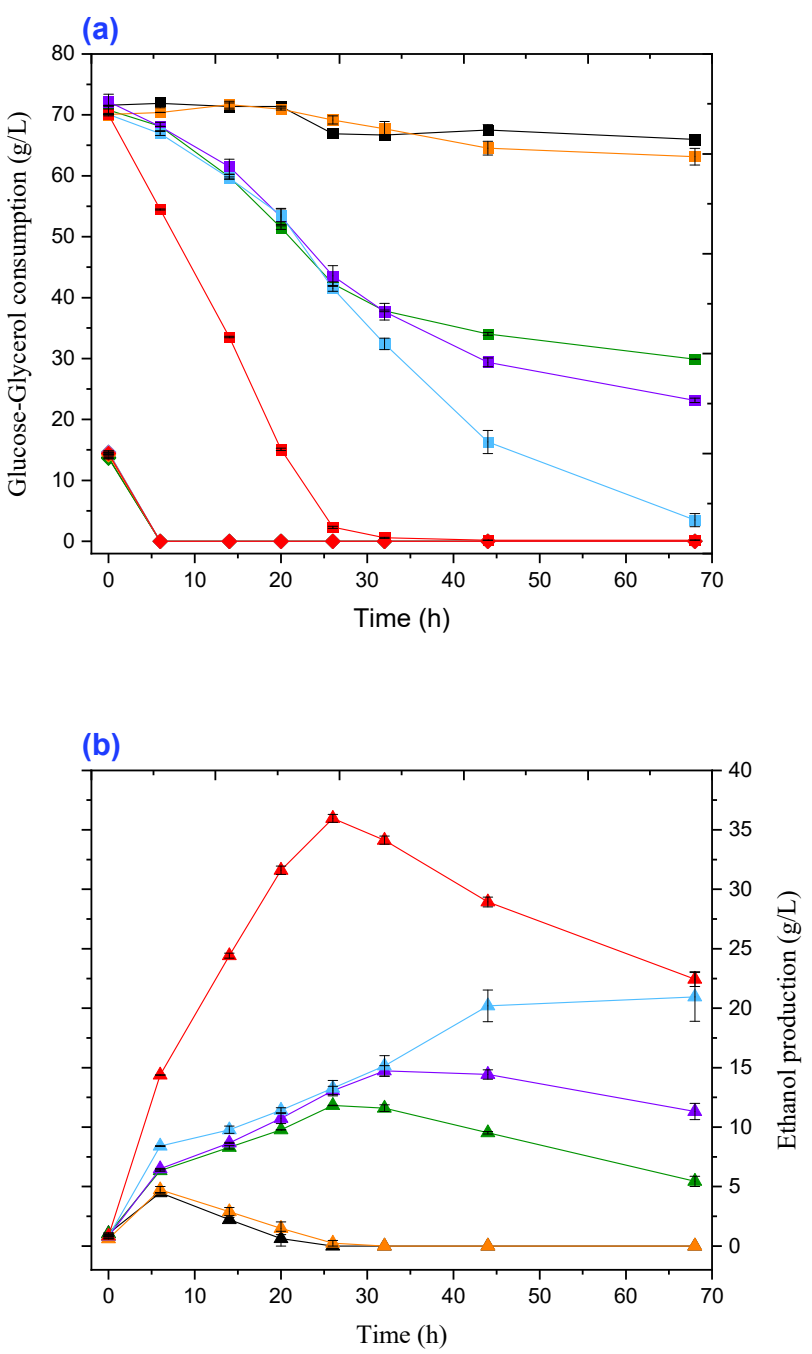

1073

1074 
1075 Fig. 3. Comparison of the time course of glycerol-glucose fermentation between the $S$. 1076 cerevisiae strains within this study: ancestral strain (black lines); NoxE/GPD1 strain

1077 (orange lines); GDH strain (green lines); GN strain (purple lines); GN-FDT strain 1078 (blue lines); GN-FDT-M1[SK-FGG] (red lines); (a) Glucose consumption (rhomboid 1079 symbols), glycerol consumption (squares); (b) ethanol production (triangles). 1080 Fermentation carried out in semi-aerobic conditions in flasks with 10:100 medium: 1081 flask volume at $30^{\circ} \mathrm{C}$ with shaking at $180 \mathrm{rpm}$. Data obtained from the mean of three 1082 independent experiments run simultaneously to decrease time differences of sampling. 1083 Error bars represent the standard deviation of the mean and are not visible when 1084 smaller than the symbol size. YP medium supplemented with (w/v) $1.5 \%$ glucose, and $10857 \%$ glycerol was used. 
Table 1: Characteristics of S. cerevisiae strains generated in this study:

\begin{tabular}{|c|c|c|}
\hline $\begin{array}{l}\text { S. cerevisiae } \\
\text { strain }\end{array}$ & Relevant genotype & Reference \\
\hline D452-2 & MATa leu2 his3 ura3 can 1 & $\begin{array}{l}\text { (Hosaka et } \\
\text { al, 1992) }\end{array}$ \\
\hline GCY1 & D452-2, HIS3:: PGK promoter and terminator - ScGCY1 & this study \\
\hline GF1 & D452-2, HIS3:: PGKp\&t-ScTPI1, ScDAK2, ScDAK1, ScGCY1 & this study \\
\hline GF2 & D452-2, HIS3:: PGKp\&t-ScTPI1, ScDAK2, ScDAK1 ScGCY1, ScSTL1 & this study \\
\hline GA1 & D452-2, HIS3:: PGKp\&t-ScTPI1, ScGUT2, ScGUT1 & this study \\
\hline GA2 & D452-2, HIS3:: PGKp\&t-ScTPI1, ScGUT2, ScGUT1, ScSTL1 & this study \\
\hline GDH & D452-2, URA3:: TDH3p-OpGDH-d22-DIT1t & this study \\
\hline$\triangle \mathrm{GPD} 1$ & D452-2, $\triangle G P D 1::$ TDH3p-d22-DIT1t & this study \\
\hline $\begin{array}{l}\text { GPD1/ LINoxE } \\
\text { (NOXE) }\end{array}$ & D452-2, $\triangle G P D 1::$ TDH3p-LINoxE-d22-DIT1t & this study \\
\hline$\Delta \mathrm{GPD} 2$ & D452-2, $\triangle G P D 2::$ TDH3p-d22-DIT1t & this study \\
\hline GPD2/ L1NoxE & D452-2, $\triangle G P D 2::$ TDH3p-LINoxE-d22-DIT1t & this study \\
\hline
\end{tabular}




\begin{tabular}{|c|c|c|}
\hline$\triangle \mathrm{GPD} 1, \triangle \mathrm{GPD} 2$ & D452-2, $\triangle G P D 1::$ TDH3p-d22-DIT1t; $\triangle G P D 2:$ TDH3p-d22-DIT1t & this study \\
\hline $\begin{array}{l}\text { GPD1/ L1NoxE - } \\
\text { GPD2/ LINoxE }\end{array}$ & D452-2, $\triangle G P D 1::$ TDH3p-LINoxE-d22-DIT1t; $\triangle G P D 2:: T D H 3 p-L I N o x E-d 22-D I T 1 t$ & this study \\
\hline $\begin{array}{ll}\text { GPD1/ L1NoxE } & - \\
\triangle \text { GPD2 } & \end{array}$ & D452-2, $\triangle G P D 1::$ TDH3p-LINoxE-d22-DIT1t; $\triangle G P D 2:: T D H 3 p-d 22-D I T 1 t$ & this study \\
\hline $\begin{array}{l}\triangle \mathrm{GPD} 1- \\
\text { GPD2/LINoxE } \\
\end{array}$ & D452-2, $\triangle G P D 1::$ TDH3p-d22-DIT1t; $\triangle G P D 2:: T D H 3 p-L I N o x E-d 22-D I T 1 \mathrm{t}$ & this study \\
\hline NoxE/URA3 & D452-2, URA3:: TDH3p-LINoxE-d22-DIT1t & this study \\
\hline $\begin{array}{l}\text { GDH-NOXE } \\
(\mathrm{GN}) \\
\end{array}$ & D452-2, URA3:: TDH3p-OpGDH-d22-DIT1t; $\triangle G P D 1::$ TDH3p-LINoxE-d22-DIT1t & this study \\
\hline $\begin{array}{l}\text { GDH-NOXE-FDT } \\
\text { (GN-FDT) }\end{array}$ & $\begin{array}{l}\text { D452-2, URA3:: TDH3p-OpGDH-d22-DIT1t; } \triangle G P D 1:: \text { TDH3p-LINoxE-d22-DIT1t; } \\
\text { AUR1-C:: PGKp-CuFPS1-RPL41Bt; PGKp-ScTPI1-PGKt; PGKp-ScDAK2-PGKt; } \\
\text { PGKp-ScDAK1-PGKt }\end{array}$ & this study \\
\hline $\begin{array}{l}\text { GN-FDT-M1 } \\
\text { (SK-FGG) }\end{array}$ & $\begin{array}{l}\text { D452-2, URA3:: TDH3p-OpGDH-d22-DIT1t; } \triangle G P D 1:: \text { TDH3p-LINoxE-d22-DIT1t; } \\
\text { AUR1-C:: PGKp-CuFPS1-RPL41Bt; PGKp-ScTPI1-PGKt; PGKp-ScDAK2-PGKt; } \\
\text { PGKp-ScDAK1-PGKt; } \quad \Delta G U T 1:: \quad \text { TEFp-CuFPS1-CYC1t; TYS1p-OpGDH-ATP15t; } \\
\text { TDH3p-ScDAK1-d22-DIT1t; FBA1p-ScTPI1-TDH3t }\end{array}$ & this study \\
\hline
\end{tabular}


1087 Table 2. Specific activities of the enzymes; glycerol dehydrogenase (GDH), dihydroxyacetone kinase (DAK), triosephosphate 1088 isomerase (TPI1) and NADH/NAD ${ }^{+}$ratio with their intracellular concentrations in the recombinant strains in this study. Error values 1089 represent standard deviation of the mean $(\mathrm{n}=2)$. * indicates not detected. $\quad$ indicates not measured.

\begin{tabular}{|c|c|c|c|c|c|c|}
\hline \multirow[t]{2}{*}{ Relevant strain } & \multicolumn{3}{|c|}{$\begin{array}{c}\text { Enzyme activities } \mu \mathrm{mole} / \mathrm{min} / \mathrm{mg} \text { cell } \\
\text { extracted proteins }\end{array}$} & \multicolumn{2}{|c|}{ Intracellular concentrations } & \multirow{2}{*}{$\begin{array}{l}\text { NADH } / \mathbf{N A D}^{+} \\
\text {ratio }\end{array}$} \\
\hline & GDH & DAKs & TPI1 & $\operatorname{NADH}(\mu \mathrm{M})$ & $\mathbf{N A D}^{+}(\boldsymbol{\mu M})$ & \\
\hline WT & ND* & $3.9 \pm 0.23$ & $0.058 \pm 0.003$ & $0.17 \pm 0.004$ & $0.36 \pm 0.001$ & $0.47 \pm 0.01$ \\
\hline GF2 & ND* & $6.4 \pm 0.05$ & $0.068 \pm 0.003$ & $0.11 \pm 0.003$ & $0.32 \pm 0.002$ & $0.35 \pm 0.01$ \\
\hline GA2 & ND* & NMa & $0.064 \pm 0.002$ & $0.26 \pm 0.005$ & $0.67 \pm 0.008$ & $0.40 \pm 0.002$ \\
\hline NOXE & ND* & NMa & $0.054 \pm 0.002$ & $0.04 \pm 0.005$ & $0.36 \pm 0.004$ & $0.12 \pm 0.014$ \\
\hline GDH & $0.063 \pm 0.001$ & NMa & $0.055 \pm 0.003$ & $0.26 \pm 0.008$ & $0.38 \pm 0.002$ & $0.68 \pm 0.025$ \\
\hline GN & $0.065 \pm 0.004$ & NMo & $0.057 \pm 0.003$ & $0.34 \pm 0.018$ & $0.35 \pm 0.009$ & $0.99 \pm 0.023$ \\
\hline GN-FDT & $0.071 \pm 0.003$ & $7.15 \pm 0.23$ & $0.067 \pm 0.004$ & $0.17 \pm 0.008$ & $0.52 \pm 0.008$ & $0.33 \pm 0.02$ \\
\hline SK-FGG & $0.111 \pm 0.002$ & $18.3 \pm 0.28$ & $0.076 \pm 0.004$ & $0.11 \pm 0.001$ & $0.63 \pm 0.008$ & $0.18 \pm 0.005$ \\
\hline
\end{tabular}


1091 Table 3. Glycerol secretion, acetic acid accumulation, ethanol production, ethanol yield rate, total glucose consumed and 1092 maximum growth (measured as optical density) after $20 \mathrm{~h}$ of fermentation of $10 \%$ glucose as the fermentable carbon source in YP 1093 medium under Micro-aerobic conditions using recombinant strains for rewriting NADH cycles via water-forming NADH oxidase. 1094 Error values represent standard deviation of the mean $(n=3)$. Micro-aerobic here indicates usage of flasks with 20:100 medium: 1095 flask volume with shaking at $150 \mathrm{rpm}$. * indicates not detected.

\begin{tabular}{|c|c|c|c|c|c|c|}
\hline Relevant strain & $\begin{array}{l}\text { Glycerol } \\
\text { secreted (g) }\end{array}$ & $\begin{array}{l}\text { Acetic } \\
\text { secreted (g) }\end{array}$ & $\begin{array}{l}\text { Ethanol } \\
\text { produced (g) }\end{array}$ & $\begin{array}{l}\text { Ethanol } \\
\text { yield g/ g } \\
\text { glucose }\end{array}$ & $\begin{array}{l}\text { Consumed } \\
\text { glucose (g) }\end{array}$ & $\begin{array}{l}\text { Optical } \\
\text { density } \\
\left(\mathrm{OD}_{600}\right)\end{array}$ \\
\hline WT & $2.56 \pm 0.06$ & $1.46 \pm 0.06$ & $42.67 \pm 0.53$ & $0.431 \pm 0.54$ & $99.01 \pm 1.660$ & $13.84 \pm 0.21$ \\
\hline$\triangle$ GPD1 & $0.47 \pm 0.07$ & $1.66 \pm 0.07$ & $45.78 \pm 1.09$ & $0.455 \pm 1.11$ & $100.58 \pm 2.20$ & $18.10 \pm 0.21$ \\
\hline$\triangle$ GPD2 & $2.08 \pm 0.05$ & $1.87 \pm 0.05$ & $44.74 \pm 0.88$ & $0.446 \pm 0.90$ & $100.18 \pm 1.10$ & $17.55 \pm 0.20$ \\
\hline$\Delta$ GPD1 + $\Delta$ GPD2 & $0.10 \pm 0.01$ & $1.85 \pm 0.03$ & $43.04 \pm 0.93$ & $0.436 \pm 0.95$ & $98.81 \pm 0.630$ & $16.81 \pm 0.17$ \\
\hline NoxE/GPD1 & $0.14 \pm 0.01$ & $2.81 \pm 0.08$ & $47.92 \pm 0.93$ & $0.474 \pm 0.96$ & $100.97 \pm 1.33$ & $16.22 \pm 0.19$ \\
\hline NoxE/GPD2 & $1.82 \pm 0.05$ & $1.96 \pm 0.06$ & $44.27 \pm 0.78$ & $0.43 \pm 0.810$ & $102.34 \pm 1.24$ & $16.53 \pm 0.27$ \\
\hline NoxE/GPD1+ NoxE/GPD2 & ND* & $3.59 \pm 0.11$ & $36.24 \pm 0.60$ & $0.429 \pm 0.62$ & $84.36 \pm 2.020$ & $11.71 \pm 0.19$ \\
\hline NoxE/GPD1+ $\Delta$ GPD2 & $0.11 \pm 0.01$ & $2.60 \pm 0.09$ & $35.25 \pm 0.61$ & $0.437 \pm 0.63$ & $80.7 \pm 2.2530$ & $13.04 \pm 0.18$ \\
\hline NoxE/GPD2+ $\Delta$ GPD1 & $0.11 \pm 0.02$ & $2.77 \pm 0.06$ & $43.25 \pm 0.86$ & $0.435 \pm 0.87$ & $99.28 \pm 2.110$ & $13.73 \pm 0.19$ \\
\hline NoxE/URA3 & $1.53 \pm 0.06$ & $2.93 \pm 0.06$ & $43.40 \pm 0.77$ & $0.43 \pm 0.820$ & $99.7 \pm 1.3230$ & $18.43 \pm 0.13$ \\
\hline
\end{tabular}


1101

Table 4. Fermentation characteristics by the generated strain SK-FGG at high concentrations of glycerol, with and without adding glucose, or with the higher availability of oxygen

\begin{tabular}{|c|c|c|c|c|c|}
\hline \multirow[b]{2}{*}{ Parameter name } & \multicolumn{5}{|c|}{ Fermentation conditions \& products values } \\
\hline & $\begin{array}{l}\text { One fed-batch } \\
{[\mathrm{A}]}\end{array}$ & $\begin{array}{l}\text { One fed-batch } \\
{[\mathrm{B}]}\end{array}$ & $\begin{array}{l}\text { Two fed-batch } \\
\text { [C] } \\
\end{array}$ & $\begin{array}{l}\text { Two fed-batch } \\
\text { [D] }\end{array}$ & $\begin{array}{l}\text { One fed-batch } \\
\text { [E] }\end{array}$ \\
\hline Oxygen availability \# & $1: 10$ & $1: 10$ & $1: 10$ & $1: 10$ & $1: 25$ \\
\hline Fermentation time (h) & $40 \pm 0.30$ & $40 \pm 0.30$ & $96 \pm 0.30$ & $96 \pm 0.30$ & $19 \pm 0.30$ \\
\hline Total consumed glycerol (g/L) & $82.5 \pm 1.63$ & $75.77 \pm 1.34$ & $141.88 \pm 2.66$ & $141.703 \pm 4.79$ & $82.38 \pm 0.34$ \\
\hline Total consumed glucose $(\mathrm{g} / \mathrm{L})$ & NA & $22.55 \pm 1.14$ & $22.2 \pm 0.67$ & $44.67 \pm 0.9$ & $16.36 \pm 0.96$ \\
\hline Rate of consumption $\left(\mathrm{gL}^{-1} \mathrm{~h}^{-1}\right)$ & $2.06 \pm 0.017$ & $2.42 \pm 0.014$ & $1.71 \pm 0.011$ & $1.94 \pm 0.015$ & $5.19 \pm 0.05$ \\
\hline Ethanol yield (g/L) & $40.42 \pm 0.89$ & $48.22 \pm 1.16$ & $79.74 \pm 1.03$ & $86.54 \pm 2.48$ & $40.93 \pm 1.21$ \\
\hline Rate of ethanol production $\left(\mathrm{gL}^{-1} \mathrm{~h}^{-1}\right)$ & $1.01 \pm 0.01$ & $1.2 \pm 0.02$ & $0.83 \pm 0.01$ & $0.9 \pm 0.02$ & $2.15 \pm 0.06$ \\
\hline Efficiency of ethanol production $\left(\mathrm{g}^{\mathrm{e}} / \mathrm{g}^{\mathrm{s}}\right)$ * & $0.49 \pm 0.002$ & $0.49 \pm 0.002$ & $0.486 \pm 0.003$ & $0.464 \pm 0.003$ & $0.414 \pm 0.01$ \\
\hline Efficient/ theoretical (\%) ${ }^{+}$ & 98 & 98 & 97.2 & 92.8 & 82.8 \\
\hline Acetic acid accumulation (g/L) & $1.14 \pm 0.02$ & $1.12 \pm 0.02$ & $1.67 \pm 0.1$ & $2.46+0.02$ & $1.6 \pm 0.19$ \\
\hline Total conversion $\left(\mathrm{g}^{\mathrm{p} /} \mathrm{g}^{\mathrm{s}}\right)^{++}$ & $0.5 \pm 0.002$ & $0.5 \pm 0.003$ & $0.496 \pm 0.005$ & $0.478 \pm 0.004$ & $0.43 \pm 0.006$ \\
\hline Cell Density $\left(\mathrm{OD}_{600}\right)$ & $10.6 \pm 0.2$ & $11.6 \pm 0.2$ & $11.6 \pm 0.3$ & $15.2 \pm 0.3$ & $14.7 \pm 0.3$ \\
\hline
\end{tabular}


1102 \#; Oxygen availability in (liquid medium: flask volume) at $30^{\circ} \mathrm{C}$ with shaking at $200 \mathrm{rpm}$.

$1103 \quad\left(\mathrm{~g}^{\mathrm{e}} / \mathrm{g}^{\mathrm{s}}\right) *$; gram ethanol per gram of glycerol.

$1104 \quad\left(\mathrm{~g}^{\left.\mathrm{p} / \mathrm{g}^{\mathrm{s}}\right)}\right)^{++}$; gram products per gram substrate

1105 Efficient/ theoretical (\%) ${ }^{+} ; \mathrm{C}_{6} \mathrm{H}_{2} \mathrm{O}_{6} \rightarrow 2 \mathrm{C}_{2} \mathrm{H}_{5} \mathrm{OH}+2 \mathrm{CO}_{2} \rightarrow$ Eq. 1

1106

(g) $\rightarrow \quad 0.51 \mathrm{~g} \quad+0.49 \mathrm{~g}$

1107

$\mathrm{C}_{3} \mathrm{H}_{8} \mathrm{O}_{3}+1 / 2 \mathrm{O}_{2} \rightarrow \mathrm{C}_{2} \mathrm{H}_{5} \mathrm{OH}+\mathrm{CO}_{2}+\mathrm{H}_{2} \mathrm{O} \rightarrow$ Eq. 2

1108

(g)

$0.5 \mathrm{~g}+0.478 \mathrm{~g}$ 
1109 Table 5. Fermentation characteristics of glycerol as a sole carbon source by the generated strain SK-FGG at different oxygen

1110 availabilities.

\begin{tabular}{|c|c|c|c|c|}
\hline \multirow{2}{*}{$\begin{array}{l}\text { Parameter name } \\
\text { Oxygen availability\# }\end{array}$} & \multicolumn{4}{|c|}{ Fermentation conditions \& products values } \\
\hline & Strict anaerobic & $\begin{array}{l}\text { Micro-aerobic } \\
(1: 5)\end{array}$ & $\begin{array}{l}\text { Semi-aerobic } \\
(1: 10)\end{array}$ & $\begin{array}{l}\text { Aerobic } \\
(1: 15) \\
\end{array}$ \\
\hline Fermentation time (h) & $96 \pm 0.30$ & $60 \pm 0.30$ & $48 \pm 0.30$ & $36 \pm 0.30$ \\
\hline Total consumed glycerol (g/L) & $0.00 \pm 0.45$ & $37.17 \pm 2.66$ & $54.01 \pm 4.88$ & $46.45 \pm 0.74$ \\
\hline Rate of consumption $\left(\mathrm{gL}^{-1} \mathrm{~h}^{-1}\right)$ & $0.0 \pm 0.0$ & $0.62 \pm 0.4$ & $1.12 \pm 0.037$ & $1.29 \pm 0.035$ \\
\hline Total ethanol produced $(\mathrm{g} / \mathrm{L})$ & $0.0 \pm 0.0$ & $15.71 \pm 1.87$ & $20.97 \pm 1.25$ & $17.97 \pm 0.53$ \\
\hline Rate of ethanol production $\left(\mathrm{gL}^{-1} \mathrm{~h}^{-1}\right)$ & $0.0 \pm 0.0$ & $0.25 \pm 0.04$ & $0.44 \pm 0.02$ & $0.5 \pm 0.01$ \\
\hline Efficiency of ethanol production $\left(\mathrm{g}^{\mathrm{e}} / \mathbf{g}^{\mathrm{s}}\right)$ * & $0.0 \pm 0.0$ & $0.42 \pm 0.05$ & $0.39 \pm 0.01$ & $0.39 \pm 0.01$ \\
\hline Efficient/ theoretical (\%) ${ }^{+}$ & 0 & 84 & 78 & 78 \\
\hline Acetic acid accumulation (g/L) & $0.0 \pm 0.0$ & $0.87 \pm 0.21$ & $2.88 \pm 0.19$ & $3.04 \pm 0.09$ \\
\hline Total conversions $\left(\mathrm{g}^{\mathrm{p}} / \mathrm{g}^{\mathrm{s}}\right)^{++}$ & $0.0 \pm 0.0$ & $0.45 \pm 0.04$ & $0.44 \pm 0.01$ & $0.45 \pm 0.01$ \\
\hline Cell density $\left(\mathrm{OD}_{600}\right)$ & $4.66 \pm 0.1$ & $8.55 \pm 0.27$ & $8.55 \pm 0.1$ & $9 \pm 0.1$ \\
\hline
\end{tabular}


1113 \#; Oxygen availability in (liquid medium: flask volume) at $30^{\circ} \mathrm{C}$ with shaking at $200 \mathrm{rpm}$.

$1114 \quad\left(\mathrm{~g}^{\mathrm{e}} / \mathrm{g}^{\mathrm{s}}\right) *$; gram ethanol per gram of glycerol.

$1115 \quad\left(\mathrm{~g}^{\mathrm{p}} / \mathrm{g}^{\mathrm{s}}\right){ }^{++} ;$gram products per gram substrate

1116 Efficient/ theoretical (\%) ${ }^{+} ; \mathrm{C}_{6} \mathrm{H}_{2} \mathrm{O}_{6} \rightarrow 2 \mathrm{C}_{2} \mathrm{H}_{5} \mathrm{OH}+2 \mathrm{CO}_{2} \rightarrow$ Eq. 1

1117

(g) $\rightarrow \quad 0.51 \mathrm{~g} \quad+0.49 \mathrm{~g}$

1118

$\mathrm{C}_{3} \mathrm{H}_{8} \mathrm{O}_{3}+1 / 2 \mathrm{O}_{2} \rightarrow \mathrm{C}_{2} \mathrm{H}_{5} \mathrm{OH}+\mathrm{CO}_{2}+\mathrm{H}_{2} \mathrm{O} \rightarrow$ Eq. 2

1119

(g)

$0.5 \mathrm{~g}+0.478 \mathrm{~g}$

1120 